\title{
Haplotype block analysis of an Argentinean hexaploid wheat collection and GWAS for yield components and adaptation
}

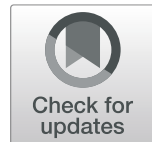

Silvana Marisol Luján Basile ${ }^{1 \dagger}$, Ignacio Abel Ramírez ${ }^{2 \dagger}$, Juan Manuel Crescente ${ }^{3,4}$, Maria Belén Conde $^{3}$, Melina Demichelis ${ }^{3}$, Pablo Abbate ${ }^{2}$, William John Rogers ${ }^{1,4}$, Ana Clara Pontaroli ${ }^{2,4}$, Marcelo Helguera ${ }^{3}$ and Leonardo Sebastián Vanzetti ${ }^{3,4^{*}}$ (D)

\begin{abstract}
Background: Increasing wheat (Triticum aestivum L.) production is required to feed a growing human population. In order to accomplish this task a deeper understanding of the genetic structure of cultivated wheats and the detection of genomic regions significantly associated with the regulation of important agronomic traits are necessary steps. To better understand the genetic basis and relationships of adaptation and yield related traits, we used a collection of 102 Argentinean hexaploid wheat cultivars genotyped with the 35k SNPs array, grown from two to six years in three different locations. Based on SNPs data and gene-related molecular markers, we performed a haplotype block characterization of the germplasm and a genome-wide association study (GWAS).

Results: The genetic structure of the collection revealed four subpopulations, reflecting the origin of the germplasm used by the main breeding programs in Argentina. The haplotype block characterization showed 1268 blocks of different sizes spread along the genome, including highly conserved regions like the 1BS chromosome arm where the 1BL/1RS wheat/rye translocation is located. Based on GWAS we identified ninety-seven chromosome regions associated with heading date, plant height, thousand grain weight, grain number per spike and fruiting efficiency at harvest (FEh). In particular FEh stands out as a promising trait to raise yield potential in Argentinean wheats; we detected fifteen haplotypes/markers associated with increased FEh values, eleven of which showed significant effects in all three evaluated locations. In the case of adaptation, the Ppd-D1 gene is consolidated as the main determinant of the life cycle of Argentinean wheat cultivars.

Conclusion: This work reveals the genetic structure of the Argentinean hexaploid wheat germplasm using a wide set of molecular markers anchored to the Ref Seq v1.0. Additionally GWAS detects chromosomal regions (haplotypes) associated with important yield and adaptation components that will allow improvement of these traits through marker-assisted selection.
\end{abstract}

Keywords: Wheat, Haplotype, GWAS, Heading date, Plant height, Thousand grain weight, Grain number per spike, Fruiting efficiency at harvest

\footnotetext{
*Correspondence: vanzetti.leonardo@inta.gob.ar

† Silvana Marisol LujánBasile and Ignacio Abel Ramirez contributed equally to this work.

${ }^{3}$ Laboratorio de Biotecnología, EEA INTA Marcos Juárez, Grupo Biotecnología y

Recursos Genéticos, Instituto Nacional de Tecnología Agropecuaria, Ruta 12

s/n, 2580 Marcos Juárez, Argentina

${ }^{4}$ Consejo Nacional de Investigaciones Científicas y Técnicas (CONICET).,

Buenos Aires, Argentina

Full list of author information is available at the end of the article
}

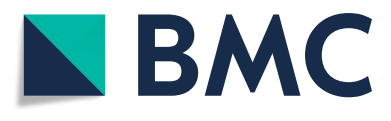

(C) The Author(s). 2019 Open Access This article is distributed under the terms of the Creative Commons Attribution 4.0 International License (http://creativecommons.org/licenses/by/4.0/), which permits unrestricted use, distribution, and reproduction in any medium, provided you give appropriate credit to the original author(s) and the source, provide a link to the Creative Commons license, and indicate if changes were made. The Creative Commons Public Domain Dedication waiver (http://creativecommons.org/publicdomain/zero/1.0/) applies to the data made available in this article, unless otherwise stated. 


\section{Background}

World population is projected to grow to nearly 10 billion by 2050 and more than 11 billion by 2100 and, on a global scale, agriculture expansion has slowed down and production increases have been achieved mainly through agricultural intensification [1]. Satisfying the increasing feed and food demand will mainly come from yield improvement: it will be required that wheat yield (and other staple crops) be increased by at least $50 \%$ in the next few decades [2], which will depend, among other components, on improving yield potential [3]. This scenario becomes more complex if we consider that each degree-Celsius increase in global mean temperature would, on average, reduce global and in particular Argentinean wheat yields by $6.0 \%[4,5]$. Furthermore, crop management will need to be environmentally more sustainable in the future [1]. Under this restrictive context, yield genetic gain will be required to increase by $1.16-1.31 \%$ per year to satisfy the projected demand of cereals for food, feed and biofuels by 2050 [6]. Unfortunately, genetic gains reported in wheat from different countries for the last decades seem to have been increasing less than required [7-9].

In Argentina, with 4.46 Mha, wheat is the third most important crop in terms of planted area after soybean and corn, average 2012-2016 [10], spanning a wide range of environments. Since 1999 the genetic gain of local cultivars has shown signs of stagnation, as indicated by genetic gain values of only $0.18 \%$ per year, mostly explained by a stabilization in grain number per unit area without changes in grain weight. Modern cultivars have increased the number of grains per spike without changes in spike number per unit area; fruiting efficiency (a.k.a. spike fertility index) was the trait that better explained the changes in grain number per unit area, and its improvement might be a way to promote increments in grain number without penalization in grain weight [11-13].

Quantitative trait locus (QTL) mapping is currently key for understanding the genetic basis of complex traits [14], including yield components and adaptation. Cost-effective, high-throughput genotyping tools for genetic study, such as single nucleotide polymorphism (SNP) arrays, are now available for all major crop species, including wheat [15], enabling the characterisation of (i) genome-wide population diversity and structure; (ii) selective sweeps and directional selection; and (iii) marker-trait relationships in genome-wide association studies (GWAS) [16, 17]. Nonetheless, in this latter context, certain limitations are found: (i) SNPs only provide biallelic information [18]; and (ii) associations are likely to be due to loci in linkage disequilibrium (LD) with a gene or a controlling sequence, rather than to underlying causal reasons [19]. To overcome the biallelic problem and increase the resolution of candidate regions, the analysis of haplotypes can be employed, i.e. of the combination of co-inherited markers from polymorphic sites within a certain chromosome region. Exploring haplotypes in this way can take full advantage of ancient recombination events in order to identify the genetic loci underlying traits at a relatively high resolution [20].

The recently released wheat reference genome assembly (IWGSC Ref. Seq. v1.0), developed by the International Wheat Genome Sequencing Consortium (IWGSC) [21], allows a more accurate comparison of the chromosome regions associated with agronomic traits from independent research studies and helps accelerate cloning of further important genes for wheat breeding.

In this study, 102 Argentinean wheat cultivars were genotyped by using the 35k Axiom Wheat Breeder's Genotyping Array [22]. The polymorphic SNPs were physically anchored to the wheat reference genome assembly [21] and the genetic structure of the collection was determined. Based on multi-environmental trial data, haplotype-based GWAS was conducted to identify chromosome regions affecting crop adaptation and the building of yields. Adaptation traits include heading date, a relevant trait since it will determine the environment to which the crop will be exposed during its reproductive stages, and plant height, which is the trait most associated with lodging in Argentinean cultivars [23]. Yieldrelated traits include thousand grain weight and number of grains per spike, currently called "numerical components of yield", which are the main factors proposed by [24] to analyze the differences in yield between cultivars. Additionally, [25] proposed considering the number of grains (GN) as the product between two variables currently known as "ecophysiological components": the spike dry weight at anthesis (SDWA) and the fruiting efficiency (FE, aka spike fertility index, ie the quotient between GNS and SDWA). Later [26] proposed the use of spike dry weight at harvest without grains (or chaff) as a substitute to SDWA. Then, the fruiting efficiency is calculated in this work as the grain number produced per unit of chaff (FEh). The FEh has shown a high association with the GN in both Argentinean [13, 27-29] and foreign [30] cultivars and moderately high heritability [12, 28, 29]. GWAS provides valuable information for a better understanding of the major genetic components involved in adaptation and yield components for bread wheat, with major implications for breeding programs aimed at increasing yield potential of new cultivars, in order to contribute to food security.

\section{Results}

\section{SNP-based genetic structure of the Argentinean wheat} collection

The genotyping of the 102 Argentinean hexaploid wheat cultivars collection yielded a total of 7972 polymorphic 
SNPs with MAF $\geq 10 \%$. A set of 6486 polymorphic SNPs was genetically anchored to specific wheat chromosomes based on mapping information from five biparental populations described previously [22]. A second set of 909 additional SNPs were anchored to specific chromosomes using three local biparental populations and an additional 577 SNPs were anchored using the association method mentioned above (Additional file 1: Table S1). Of all the anchored SNPs, 3034 were on the A subgenome, 4010 on the B subgenome and the lowest number, 928 SNPs, on the D subgenome. To reveal the genetic relationships among the 102 Argentinean hexaploid wheat cultivars collection, we conducted a model-based approach using $\mathrm{R}$ STRUCTURE software [31]. A model-based approach is a cluster analysis that evaluates genetic similarity among genotypes without using prior information. For the analysis we used the 7972 SNPs anchored to the wheat genome described before. We detected four subpopulations in the collection reflecting the origin of the germplasm used by the main breeding programs in Argentina (Additional file 2: Table S2). A graphical representation of the STRUCTURE Q matrix was included (Additional file 3: Figure S1). Subpopulation 1 (Table 1) included only introduced cultivars of French origin (100\%), with the Nidera, Syngenta and Sursem breeding companies exclusively represented in this group. Subpopulation 2 included most of the old cultivars ( 9 out of 12 released between 1930 and 1990), also known as traditional germplasm [32, 33] and all cultivars from the Don Mario breeding company released up until 2010. Subpopulation 3 included cultivars released by the Klein breeding company (46.15\%) and, in lower frequency, cultivars belonging to the ACA, Buck, INTA and Relmó breeding companies, most of them with pedigrees including CIMMYT germplasm. Finally, subpopulation 4 included cultivars released by INTA (33.33\%), Buck, Klein, ACA and Relmó, with most of them also having pedigrees including CIMMYT germplasm.

\section{SNP and gene marker-based haplotype blocks construction and characterization of the Argentinean wheat cultivar collection}

A detailed information about the haplotype-based map construction for the Argentinean wheat collection is found in the Table 2. A total of 1268 haplotype blocks (HBs) were built in our wheat cultivars collection, of which 518 were on the A subgenome, 641 on the B subgenome and 109 on the D subgenome. The $94 \%$ of the SNP and gene markers were involved in these HBs and only $495 \mathrm{SNP} /$ gene-related markers were not included in any $\mathrm{HB}$. The average block sizes were significantly larger on the D subgenome chromosomes than on the $\mathrm{A}$ or B subgenomes, with observed averages of 13.5, 6.7 and $4.8 \mathrm{Mb}$, respectively (Table 2 ). In general, within each chromosome, the largest $\mathrm{HBs}(>30 \mathrm{Mb})$ were placed in the centromeric region, where recombination is lower, while the smallest HBs were located towards the telomeric regions, where the recombination rate and gene density are higher (Fig. 1a). The average distance between HBs on the D subgenome was 8 times larger than that for the B or A subgenomes, with an average of 16.1, 2.7 and $2.4 \mathrm{Mb}$, respectively. The maximum spacing between segments was also significantly higher in the $D$ subgenome with respect to $B$ and $A$, with average values of 106.6, 57.1 and 44.2 Mb, respectively (Table 2). An interesting case can be described for chromosome $1 \mathrm{~B}$, where we observed several large HBs on the short arm, from HB 9 to 20 (HB average size $24 \mathrm{Mb}$ in the region). A possible explanation is the presence of the 1BL/1RS wheat/rye translocation which is maintained at high frequency in Argentinean cultivars. The lack of recombination events within the translocation generates a highly conserved block involving almost the entire short arm of chromosome 1B (Fig. 1b) (Table 2).

\section{Broad sense heritability and correlations among traits}

All evaluated traits showed high heritability: the crop adaptation traits $\mathrm{HD}$ and $\mathrm{PH}$ showed broad sense heri-

Table 1 Distribution of the 102 Argentinean bread wheat cultivars in the four subpopulations detected using a model-based approach

\begin{tabular}{ll}
\hline Subp. & Cultivar \\
\hline 1 & Baguette 10; Baguette 17; Baguette 18; Baguette 19; Baguette 21; Baguette 30; Baguette 31; Baguette 9; Baguette P. 11; SY 100; SY 200; \\
& SY 300; SRN Nogal \\
& ACA 201; ACA 320; Barletta 77; BIOINTA 1004; BIOINTA BONAERENSE 2001; BIOINTA 2004; Buck Chacarero; Buck Guapo; Buck Malevo; \\
& Buck Mangrullo; Buck Meteoro; Buck Napostá; Buck Norteño; Buck Pingo; Buck Ranquel; Buck Taita; Don Mario Arex; Don Mario Atlax; \\
& Don Mario Cronox; Don Mario Onix; Don Mario Themix; LE 2249; LE 2271; Klein 32; Klein Atlas; Klein Centauro; Klein Escorpión; Klein \\
& Impacto; Klein Proteo; Klein Rendidor; KleinTauro; Olaeta Artillero; Sinvalocho
\end{tabular}

3 ACA 223; ACA 903B; ACA 906; BIOINTA 1001; BIOINTA 3004; Buck Baqueano; Buck Huanchen; Buck Puelche; Klein Brujo; Klein Cacique; Klein Carpincho; Klein Castor; Klein Don Enrique; Klein Gavilán; Klein Gladiador; Klein Guerrero; Klein Nutria; Klein Tigre; Klein Yarará; Klein Zorro; LE 2333; LE 2341; ProlNTA Gaucho; ProlNTA Granar; ProlNTA Guazú; ProINTA Oasis 
Table 2 Detailed information about the haplotype-based map construction for the Argentinean wheat collection

\begin{tabular}{|c|c|c|c|c|c|c|c|c|c|}
\hline \multirow{2}{*}{$\begin{array}{l}\mathrm{Chr} . \\
\mathrm{SG}^{1}\end{array}$} & \multirow{2}{*}{$\begin{array}{l}\text { SNPS/ } \\
\mathrm{GM}^{2}\end{array}$} & \multirow{2}{*}{$\begin{array}{l}\text { Haplotype } \\
\text { count }\end{array}$} & \multirow{2}{*}{$\begin{array}{l}\mathrm{HB}^{3} \\
\text { count }\end{array}$} & \multicolumn{2}{|c|}{ SNPs per HB } & \multicolumn{2}{|c|}{ HB size ${ }^{4}$} & \multicolumn{2}{|c|}{ HB spacing ${ }^{4}$} \\
\hline & & & & Avg. & Max. & Avg. & Max. & Avg. & Max. \\
\hline $1 \mathrm{~A}$ & 431 & 244 & 66 & 6.2 & 26 & 4.5 & 59.4 & 3.4 & 125.4 \\
\hline $1 \mathrm{~B}$ & 919 & 327 & 91 & 9.7 & 168 & 5.8 & 115.6 & 1.3 & 21.6 \\
\hline $1 \mathrm{D}$ & 253 & 74 & 22 & 10.8 & 90 & 8.5 & 48.8 & 10.0 & 115.8 \\
\hline $2 \mathrm{~A}$ & 421 & 239 & 70 & 5.4 & 60 & 7.9 & 166.7 & 2.1 & 26.7 \\
\hline $2 B$ & 709 & 459 & 127 & 5.2 & 40 & 3.8 & 88.8 & 1.8 & 35.9 \\
\hline $2 \mathrm{D}$ & 272 & 79 & 23 & 11.4 & 38 & 16.7 & 240.6 & 9.0 & 91.1 \\
\hline $3 \mathrm{~A}$ & 389 & 268 & 75 & 4.8 & 22 & 6.7 & 261.7 & 2.3 & 28.6 \\
\hline $3 B$ & 597 & 421 & 116 & 4.8 & 18 & 3.5 & 40.3 & 2.7 & 101.3 \\
\hline $3 \mathrm{D}$ & 115 & 56 & 15 & 7.4 & 21 & 21.8 & 124.5 & 16.9 & 80.7 \\
\hline $4 \mathrm{~A}$ & 326 & 216 & 59 & 5.1 & 36 & 9.5 & 211.3 & 2.2 & 16.3 \\
\hline $4 \mathrm{~B}$ & 253 & 141 & 42 & 5.3 & 26 & 4.7 & 39.1 & 6.6 & 162.4 \\
\hline $4 \mathrm{D}$ & 47 & 29 & 8 & 4.1 & 14 & 16.0 & 78.3 & 17.6 & 103.2 \\
\hline $5 \mathrm{~A}$ & 544 & 340 & 99 & 5.3 & 38 & 4.6 & 247.9 & 2.1 & 49.8 \\
\hline $5 B$ & 641 & 365 & 102 & 5.8 & 67 & 4.5 & 110.1 & 1.7 & 19.7 \\
\hline $5 D$ & 110 & 36 & 11 & 9.3 & 28 & 13.7 & 133.7 & 21.6 & 106.2 \\
\hline $6 \mathrm{~A}$ & 433 & 227 & 60 & 7.0 & 55 & 8.2 & 168.9 & 2.5 & 33.5 \\
\hline $6 B$ & 532 & 300 & 86 & 5.8 & 55 & 5.6 & 202.1 & 2.0 & 21.1 \\
\hline $6 \mathrm{D}$ & 69 & 45 & 13 & 4.8 & 12 & 4.6 & 32.1 & 20.6 & 153.5 \\
\hline $7 \mathrm{~A}$ & 509 & 330 & 89 & 5.3 & 34 & 5.2 & 121.4 & 2.3 & 28.8 \\
\hline $7 \mathrm{~B}$ & 374 & 270 & 77 & 4.5 & 18 & 6.0 & 89.2 & 2.9 & 37.8 \\
\hline $7 D$ & 80 & 50 & 17 & 4.2 & 11 & 13.2 & 185.0 & 16.9 & 95.7 \\
\hline A & 436.1 & 266.3 & 74 & 5.6 & 38.7 & 6.7 & 176.8 & 2.4 & 44.2 \\
\hline B & 575.0 & 326.1 & 92 & 5.9 & 56.0 & 4.8 & 97.9 & 2.7 & 57.1 \\
\hline D & 82.1 & 52.7 & 16 & 7.5 & 30.6 & 13.5 & 120.4 & 16.1 & 106.6 \\
\hline Overall & 436.9 & 269.5 & 60 & 6.3 & 41.6 & 8.3 & 131.7 & 7.1 & 69.3 \\
\hline
\end{tabular}

$1 \mathrm{SG}=$ Subgenomes. ${ }^{2} \mathrm{GM}=$ Gene-related Markers. ${ }^{3} \mathrm{HB}=$ Haplotype Blocks. ${ }^{4}$ The units are expressed in Mb based on the coordinates in the IWGSC Ref. Seq. 1.0 wheat genome assembly

tability values higher than 0.95 and the yield related traits FEh, TGW and GNS displayed values in all cases higher than 0.80 (Table 3).

Regarding correlations between traits across the tested locations, the most consistent pattern was observed for FEh vs GNS, with positive correlations in most years at the Southern locations, Azul and Balcarce, and in all years at the Northern location Marcos Juárez (Table 4). The relationship between FEh vs TGW showed no significant correlations in all tested environments, except Azul 2014.

The $\mathrm{PH}$ showed a positive correlation with TGW in Azul (one year) and in all years in Marcos Juárez with higher significant values. On the other hand, the $\mathrm{PH}$ showed negative correlations with GNS in most years at the Southern locations, contrasting with Marcos Juárez with no significant correlation in most tested years. A similar pattern can be described for $\mathrm{PH}$ vs FEh, with negative correlations in most years at the Southern locations, contrasting with Marcos Juárez with no significant correlations in all years (Table 4).

The HD showed a positive correlation with GNS in the Southern locations (2 years), contrasting with Marcos Juárez with negative correlations in three years. The HD also showed negative and highly significant correlations with TGW in Azul (1 year) and Marcos Juárez (2 years). A positive correlation between HD and FEh was observed in Azul (2 years), differing from Marcos Juárez with no significant correlations in most tested years. The HD vs PH showed contrasting correlations at the Southern locations and no significant correlations in most years in Marcos Juárez. The TGW vs GNS correlation showed positive values in Marcos Juárez (3 years), with no significant correlations in most Southern environments (Table 4).

Contrasting correlations observed across the Southern and Northern locations for PH vs GNS, PH vs FEh, 


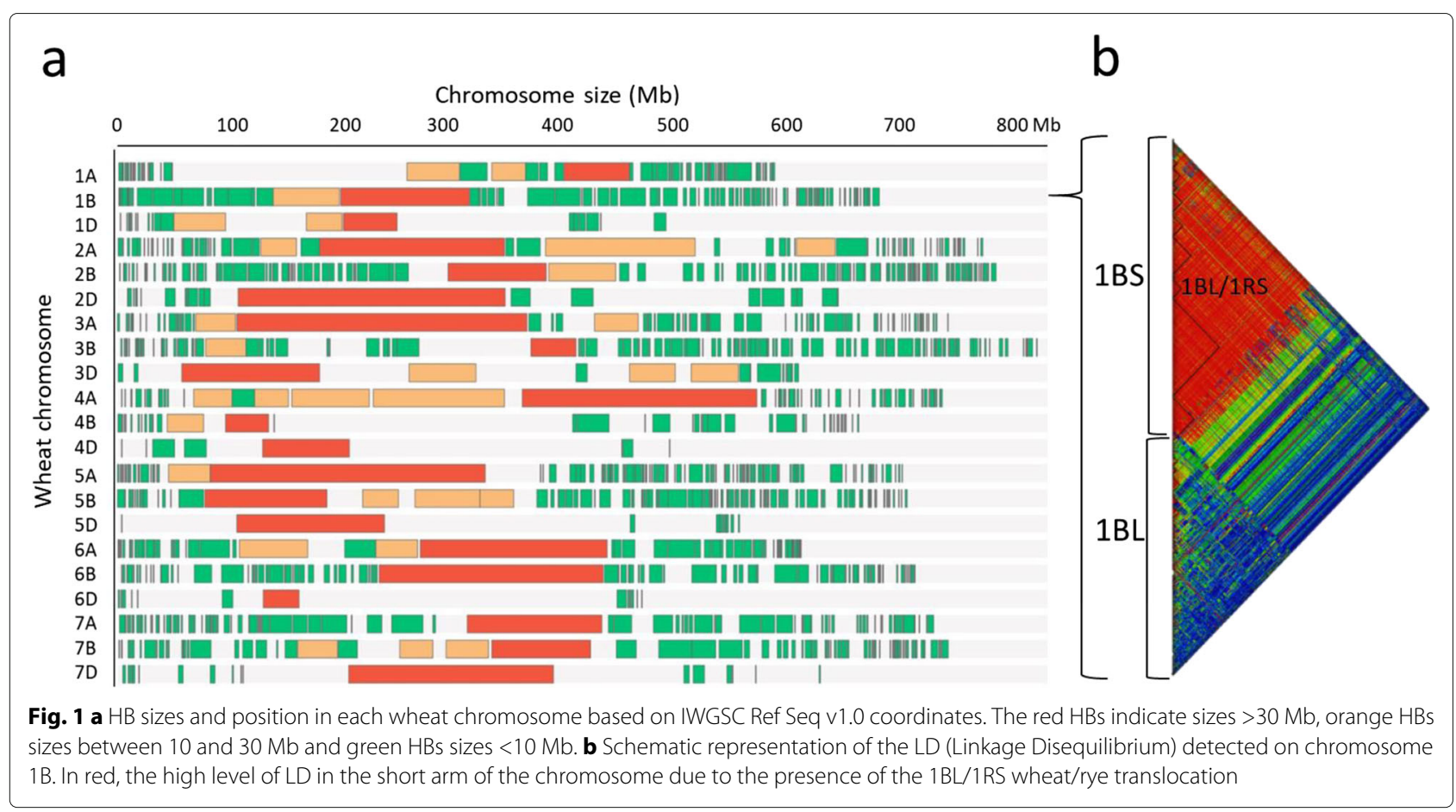

HD vs GNS, HD vs FEh and, to a lesser extent, PH vs TGW, might suggest different strategies are involved in building site-specific yield components performance: genotypes adapted to the Northern latitudes would be favored by taller plants improving TGW, but affected by late flowering penalyzing TGW and GNS, whereas genotypes adapted to Southern latitudes would be favored by late flowering improving GNS and FEh, but affected by taller plants penalyzing FEh. Interestingly, independently of the environment, genotypes with high FEh would favor GNS, one of the most important yield components.

\section{Haplotype-based gWAS analysis}

Fruiting efficiency at harvest (FEh):

We identified 17 haplotypes/markers associated with FEh of which one belongs to chromosome $1 \mathrm{~A}$, seven to $2 \mathrm{~A}$,

Table 3 Broad sense heritability $\left(H^{2}\right)$ and variance components for the best linear unbiased predictors (BLUPS) of five traits across the tested environments

\begin{tabular}{llllll}
\hline Trait & No. env. & $\sigma_{G}^{2}$ & $\sigma_{E}^{2}$ & $\sigma_{e}^{2}$ & $H^{2}$ \\
\hline FEh & 10 & 74.69 & 232.24 & 139.43 & 0.84 \\
TGW & 10 & 5.24 & 20.19 & 12.63 & 0.81 \\
GNS & 10 & 17.43 & 28.59 & 34.77 & 0.83 \\
HD & 11 & 89.31 & 143.44 & 39.76 & 0.96 \\
PH & 11 & 79.94 & 109.18 & 26.52 & 0.97 \\
\hline
\end{tabular}

$\sigma_{G}^{2}=$ genotypic variance; $\sigma_{E}^{2}=$ environmental variance; $\sigma_{e}^{2}=$ residual variance (here $=\sigma_{G \times E}^{2}$ since BLUPs have a single replication per environment); $H^{2}=$ broad sense heritability. All genotype and environment variances were signifcant at $P<0.001$ one to $3 \mathrm{~B}$, two to $4 \mathrm{~A}$, two to $5 \mathrm{~A}$, three to $6 \mathrm{~A}$ and one to 7A (Table 5; Additional file 6: Table S5). Eleven haplotypes associated with FEh were significant in at least one year in each of the three locations, i.e. Southern (Azul and Balcarce) and Northern (Marcos Juárez). Five haplotypes were significant exclusively in Southern locations and only the SNP AX-94874921 located on chromosome $2 \mathrm{~A}(707.1 \mathrm{Mb})$ showed significant association exclusively in Marcos Juárez.

An example of a haplotype with significant association with FEh across Southern and Northern locations is Chr5A-B43-Hap1, located on chromosome 5A (476.4$476.7 \mathrm{Mb}$ ) (Fig. 2a). The HB Chr5A-B43 is composed of seven SNPs, and the significantly associated haplotype Hap1.The Chr5A-B43-Hap1 was detected in 16 out of 102 evaluated cultivars (Fig. 2b). The presence of Chr5AB43-Hap1 significantly increased FEh in the three tested locations, albeit with different intensities, with a stronger effect in Southern locations (Fig. 2c).

Three haplotypes significantly associated with FEh were detected as colocating with additional traits: haplotype Chr2A-B49-Hap2 on 2A (704.8-705.8 Mb) was colocated with GNS, haplotype Chr5A-B33-Hap4 on chromosome 5A (445.2-445.2 Mb) with PH and Chr6A-B24-Hap2 on chromosome 6A (205.1-233.3 Mb) with TGW. The correlations for the first two haplotypes were significant only in Southern locations (Azul and Balcarce), while the third was significant in all locations. In the case of Chr2A-B49Hap2, the correlation with GNS was positive, as shown in Table 5, and the cultivars with the haplotype for higher 
Table 4 Pearson's correlation among traits in the different tested environments

\begin{tabular}{|c|c|c|c|c|c|c|c|c|c|}
\hline \multirow[b]{3}{*}{ Traits } & \multicolumn{4}{|c|}{ South locations } & \multirow{2}{*}{\multicolumn{5}{|c|}{$\begin{array}{l}\text { North location } \\
\text { Marcos Juárez }\end{array}$}} \\
\hline & \multicolumn{2}{|l|}{ Azul } & \multicolumn{2}{|l|}{ Balcarce } & & & & & \\
\hline & $13^{1}$ & 14 & 14 & 15 & 12 & 13 & 14 & 15 & 16 \\
\hline HDvsPH & $-0.23^{*}$ & ns & $0.26^{*}$ & $0.32^{*}$ & ns & ns & $0.35^{* *}$ & ns & ns \\
\hline HDvsFEh & $0.23^{*}$ & $0.52^{* * *}$ & ns & ns & ns & ns & ns & ns & $-0.31^{*}$ \\
\hline HDvsTGW & ns & $-0.42^{* * *}$ & ns & ns & ns & ns & ns & $-0.47^{* * *}$ & $-0.34^{* *}$ \\
\hline HDvsGNS & ns & $0.44^{* * *}$ & $0.26^{*}$ & ns & ns & $-0.31^{*}$ & ns & $-0.41^{* * *}$ & $-0.44^{* * *}$ \\
\hline PHvsFEh & $-0.28^{*}$ & ns & $-0.32^{*}$ & $-0.35^{* *}$ & ns & ns & ns & ns & ns \\
\hline PHvsTGW & ns & $0.24^{*}$ & ns & ns & $0.48^{* * *}$ & $0.37^{* *}$ & $0.40^{* * *}$ & $0.39^{* * *}$ & $0.43^{* * *}$ \\
\hline PHvsGNS & $-0.23^{*}$ & ns & $-0.38^{* * *}$ & $-0.31^{*}$ & $0.21^{*}$ & ns & ns & ns & ns \\
\hline FEhvsTGW & ns & $-0.67^{* * *}$ & ns & ns & ns & ns & ns & ns & ns \\
\hline FEhvsGNS & ns & $0.47^{* * *}$ & $0.35^{* *}$ & $0.24^{*}$ & $0.58^{* * *}$ & $0.31^{*}$ & $0.38^{* *}$ & $0.36^{* *}$ & $0.58^{* * *}$ \\
\hline TGWvsGNS & $0.22^{*}$ & $-0.21^{*}$ & ns & ns & ns & ns & $0.25^{*}$ & $0.48^{* * *}$ & $0.43^{* * *}$ \\
\hline
\end{tabular}

${ }^{1}$ years after 2000; ns $=$ not significant, ${ }^{*} P<0.05,{ }^{* *} P<0.001,{ }^{* * *} P<0.0001$. HD, heading date; $\mathrm{PH}$, plant height; FEh, fruiting efficiency at harvest; TGW, thousand grain weight; GNS, grains number per spike

FEh also presented higher GNS. In the case of Chr5AB33-Hap4, the correlation with PH was negative (Table 5) and cultivars with the haplotype for higher FEh values showed lower values for PH. Finally, for Chr6A-B24-Hap2, the correlation was also negative (Table 5): cultivars with the haplotype for higher FEh values showed lower values for TGW.

\section{Thousand grain weight (TGW):}

We identified 11 haplotypes/markers associated with TGW, of which one belongs to chromosome 2D, two to $3 \mathrm{~A}$, one to $3 \mathrm{~B}$, four to $6 \mathrm{~A}$ and three to $7 \mathrm{~B}$ (Table 5). The SNP AX-95257035 located on chromosome 3A (686.8 $\mathrm{Mb}$ ) was significantly associated with TGW only in Marcos Juárez in four of five tested years. The remaining ten haplotypes/markers showed significant associations with TGW across Southern and Northern locations with, in general terms, higher significance values at the Northern location (Table 5).

Two haplotypes significantly associated with TGW and colocating with other traits were detected. The Chr3BB111-246 Hap4 on chromosome 3B (817.4-817.8 Mb) colocated with $\mathrm{PH}$, with the previously described Chr6AB24-247 Hap2 on chromosome 6A (205.1-233.3Mb) colocating with FEh. In the case of Chr3B-B111-Hap4, the correlation with $\mathrm{PH}$ was positive, as cultivars carrying the haplotype for higher TGW also expressed higher PH (Table 5).

\section{grain number per spike (GNS):}

We identified two haplotypes associated with GNS, one belonging to chromosome $2 \mathrm{~A}$ and the second to $4 \mathrm{~A}$ (Table 5). The Chr2A-B49-Hap2 was significantly associated across all locations and Chr4A-B19-Hap5 was significantly associated in Azul and Marcos Juárez, though with no significant effect in Balcarce. The haplotype Chr2AB49-Hap2 colocated with FEh and its effect was previously described (Table 5).

\section{Heading date $(H D)$ :}

A total of 16 haplotypes/markers showed significant associations with $\mathrm{HD}$. The regions were distributed as follows: three on chromosome $1 \mathrm{~B}$, two on $2 \mathrm{~B}$, one on $2 \mathrm{D}$, four on $3 \mathrm{~A}$, one on $3 \mathrm{D}$, one on $5 \mathrm{~B}$, two on $6 \mathrm{~A}$, one on $6 \mathrm{~B}$ and one on chromosome 7A. For this trait, it is important to highlight the consistent effect of the functional marker for the Ppd-D1 gene on chromosome 2D, which showed significant associations over the three tested locations during in all evaluated years. The highest associations were detected in Marcos Juárez with latitude $5^{\circ}$ lower than Southern locations (Azul and Balcarce). Cultivars carrying the Ppd-D1 sensitive allele (42 of the 102 cultivars tested) delayed flowering in all environments. Also it is important to mention that all haplotypes affecting HD showed significant associations at the Northern location in two or more years, contrasting with Southern locations Azul, showing three haplotypes with no significant effects on HD, and Balcarce, with nine haplotypes in the same situation. Additionally, we identified one haplotype colocated with PH, Chr6B-B26-Hap3 (157.6-157.8Mb), that increased HD values and negatively affected PH (Table 5).

\section{Plant height (PH):}

In the haplotype-based GWAS, 51 chromosome regions were associated with this trait. Among these, one was located on chromosome $1 \mathrm{~A}$, three on $1 \mathrm{~B}$, three on $1 \mathrm{D}$, two on $2 \mathrm{~A}$, eight on $2 \mathrm{~B}$, three on $3 \mathrm{~A}$, five on $3 \mathrm{~B}$, one on $4 \mathrm{~A}$, two on $4 \mathrm{~B}$, four on $5 \mathrm{~A}$, three on $5 \mathrm{~B}$, six on $6 \mathrm{~A}$, seven on $6 \mathrm{~B}$, one on $7 \mathrm{~A}$ and two on chromosome 7B. Forty- 
Table 5 GWAS results for FEh, TGW, GNS, HD and PH. Information of haplotypes/SNPs physical location, frequency in the collection and effect direction, $P$ values in each environment and the colocated traits

\begin{tabular}{|c|c|c|c|c|c|c|c|c|c|c|c|c|c|c|c|c|c|}
\hline \multirow[b]{2}{*}{ Trait } & \multirow[b]{2}{*}{ Haplotype/SNP 1} & \multicolumn{3}{|c|}{ Ref. Seq. v1.0 (Mb) } & \multirow{2}{*}{$\begin{array}{l}\text { Freq. } \\
\text { Effect }^{2}\end{array}$} & \multicolumn{2}{|c|}{ Azul } & \multicolumn{3}{|c|}{ Balcarce } & \multicolumn{6}{|c|}{ Marcos Juárez } & \multirow[b]{2}{*}{ Coloc. } \\
\hline & & Chr. & Start & End & & $13^{3}$ & 14 & 13 & 14 & 15 & 11 & 12 & 13 & 14 & 15 & 16 & \\
\hline \multirow[t]{17}{*}{ FEh } & Chr1A-B32-Hap4 & $1 \mathrm{~A}$ & 482.3 & 487.0 & $6+$ & * & ns & * & * & $* *$ & - & ns & ns & ns & ns & ns & \\
\hline & Chr2A-B19-Hар3 & $2 \mathrm{~A}$ & 70.2 & 72.9 & $12+$ & * & ns & * & $*$ & ns & - & $* *$ & ns & * & ns & ns & \\
\hline & Chr2A-B23-Hap1 & $2 \mathrm{~A}$ & 83.8 & 84.5 & $52-$ & * & * & * & * & ns & - & * & ns & ns & * & * & \\
\hline & Chr2A-B26-Hар3 & $2 \mathrm{~A}$ & 102.0 & 102.7 & $36+$ & * & $* *$ & ** & $*$ & ns & - & ns & ns & ns & ns & * & \\
\hline & Chr2A-B30-Hap4 & $2 \mathrm{~A}$ & 165.8 & 182.1 & $7+$ & $* *$ & ns & $*$ & * & ns & - & ns & * & ns & ns & ns & \\
\hline & Chr2A-B49-Hap2 & $2 \mathrm{~A}$ & 704.8 & 705.8 & $15+$ & * & $*$ & * & $* *$ & * & - & ns & ns & ns & ns & ns & GNS \\
\hline & AX-94874921 & $2 \mathrm{~A}$ & 707.1 & - & $16+$ & ns & ns & ns & ns & ns & - & $* *$ & ns & * & * & * & \\
\hline & Chr2A-B55-Hap1 & $2 \mathrm{~A}$ & 716.8 & 718.9 & $12+$ & * & $* * *$ & ** & * & * & - & ns & ns & ns & ns & ns & \\
\hline & Chr3B-B14-Hap4 & $3 B$ & 23.0 & 24.0 & $6+$ & ns & ns & $* *$ & $*$ & * & - & $* *$ & ns & ns & * & ns & \\
\hline & AX-94869767 & $4 \mathrm{~A}$ & 606.4 & - & $18+$ & * & ns & * & * & ns & - & * & * & ns & * & $*$ & \\
\hline & Chr4A-B35-Hар3 & $4 \mathrm{~A}$ & 625.7 & 625.9 & $23+$ & $* *$ & * & $* *$ & * & * & - & ns & ns & ns & ns & ns & \\
\hline & Chr5A-B33-Hap4 & $5 \mathrm{~A}$ & 445.2 & 445.2 & $7+$ & $* *$ & * & * & * & ns & - & ns & ns & ns & ns & ns & $\mathrm{PH}$ \\
\hline & Chr5A-B43-Hap1 & $5 \mathrm{~A}$ & 476.4 & 476.7 & $15+$ & * & ns & * & $*$ & ns & - & ns & $* *$ & ns & $*$ & ns & \\
\hline & Chr6A-B24-Hap2 & $6 \mathrm{~A}$ & 205.1 & 233.3 & $17+$ & $* * *$ & $*$ & * & ns & ns & - & ns & ns & ns & ns & * & TGW \\
\hline & Chr6A-B28-Hap4 & $6 \mathrm{~A}$ & 448.7 & 454.6 & 9- & * & * & * & $* *$ & * & - & * & ns & ns & ns & ns & \\
\hline & Chr6A-B29-Hap2 & $6 \mathrm{~A}$ & 455.6 & 467.0 & $25+$ & $* * *$ & ns & * & $*$ & ns & - & ns & ns & * & ns & * & \\
\hline & Chr7A-B36-Hap2 & $7 \mathrm{~A}$ & 119.0 & 126.0 & $10+$ & * & ns & * & $* *$ & * & - & ns & ns & ns & ns & * & \\
\hline \multirow[t]{11}{*}{ TGW } & Chr2D-B5-Нар3 & $2 \mathrm{D}$ & 17.6 & 18.2 & 7- & * & * & ns & ns & ns & - & ns & ns & * & $*$ & $* *$ & \\
\hline & Chr3A-B12-Нар3 & $3 \mathrm{~A}$ & 46.7 & 52.6 & $5-$ & ns & * & ns & * & * & - & $* *$ & * & * & ns & ns & \\
\hline & AX-95257035 & $3 \mathrm{~A}$ & 686.8 & - & $11+$ & ns & ns & ns & ns & ns & - & * & ns & $* * *$ & $* *$ & * & \\
\hline & Chr3B-B111-Hap4 & $3 B$ & 817.4 & 817.8 & $11+$ & ns & ns & ns & * & ns & - & $* *$ & ns & * & * & ns & $\mathrm{PH}$ \\
\hline & AX-94459169 & $6 \mathrm{~A}$ & 0.6 & - & $30+$ & ns & $* *$ & * & ns & ns & - & ns & * & $* * *$ & $* * *$ & $* *$ & \\
\hline & Chr6A-B20-Hар3 & $6 \mathrm{~A}$ & 63.8 & 74.0 & $21-$ & ns & ns & * & ns & ns & - & ns & ns & * & $* *$ & $*$ & \\
\hline & Chr6A-B21-Hар3 & $6 \mathrm{~A}$ & 74.5 & 101.3 & $20-$ & ns & ns & * & ns & ns & - & ns & ns & * & $* *$ & * & \\
\hline & Chr6A-B24-Hap2 & $6 \mathrm{~A}$ & 205.1 & 233.3 & $17-$ & ns & ns & $* *$ & ns & ns & - & * & ns & * & $* * *$ & * & FEh \\
\hline & Chr7B-B38-Hap3 & $7 \mathrm{~B}$ & 626.1 & 627.6 & $5+$ & ns & $* * *$ & ** & ns & ns & - & ns & * & * & ns & $* *$ & \\
\hline & Chr7B-B60-Hap2 & $7 \mathrm{~B}$ & 702.3 & 703.7 & $27+$ & ns & ns & ns & * & ns & - & * & * & $* *$ & * & * & \\
\hline & Chr7B-B60-Нар3 & $7 \mathrm{~B}$ & 702.3 & 703.7 & $15+$ & ns & ns & * & ns & ns & - & * & * & $* *$ & $* *$ & $* *$ & \\
\hline \multirow[t]{2}{*}{ GNS } & Chr2A-B49-Hap2 & $2 \mathrm{~A}$ & 704.8 & 705.8 & $15+$ & * & ns & * & $* *$ & ** & - & ns & ns & * & ns & ns & FEh \\
\hline & Chr4A-B19-Hap5 & $4 \mathrm{~A}$ & 231.0 & 349.3 & $13+$ & * & $* *$ & ns & ns & ns & - & $* *$ & * & * & ns & $*$ & \\
\hline \multirow[t]{11}{*}{ HD } & Chr1B-B76-Hap2 & $1 \mathrm{~B}$ & 643.1 & 645.7 & $35+$ & * & $* *$ & ns & ns & ns & $* *$ & * & * & ns & $*$ & * & \\
\hline & Chr1B-B84-Hap3 & $1 \mathrm{~B}$ & 667.2 & 667.8 & $17-$ & ns & ns & ** & $* *$ & * & ns & * & * & * & ns & ns & \\
\hline & Chr1B-B89-Hap4 & $1 \mathrm{~B}$ & 676.8 & 678.0 & $6-$ & ns & ns & * & $* *$ & * & * & * & ns & * & * & ns & \\
\hline & Chr2B-B40-Hap4 & $2 \mathrm{~B}$ & 162.9 & 166.9 & $10+$ & * & $* *$ & ns & ns & ns & ns & * & ns & * & * & ns & \\
\hline & Chr2B-B62-Hap1 & $2 \mathrm{~B}$ & 564.2 & 569.0 & $10+$ & * & $* *$ & ns & ns & ns & * & ns & ns & ns & * & ns & \\
\hline & Ppd-D1 sensitive & $2 \mathrm{D}$ & 34 & - & $42+$ & * & $*$ & * & * & * & $* *$ & $* * *$ & $* *$ & $* * *$ & $* * *$ & $* *$ & \\
\hline & Chr3A-B31-Hap1 & $3 \mathrm{~A}$ & 504.8 & 507.5 & $59-$ & ns & ns & * & ns & * & $* *$ & * & * & * & * & $*$ & \\
\hline & Chr3A-B49-Hар3 & $3 \mathrm{~A}$ & 631.2 & 633.1 & $12-$ & $* *$ & ns & * & * & ns & * & * & ns & * & $* *$ & * & \\
\hline & Chr3A-B59-Hap3 & $3 \mathrm{~A}$ & 695.5 & 695.7 & $19+$ & ns & * & ns & ns & ns & $* *$ & * & $* *$ & * & * & ns & \\
\hline & Chr3A-B60-Hap4 & $3 \mathrm{~A}$ & 699.4 & 700.6 & $10+$ & ns & $* *$ & ns & ns & ns & $* *$ & * & $* * *$ & $* *$ & * & ns & \\
\hline & Chr3D-B7-Hap1 & $3 D$ & 518.2 & 560.1 & $54-$ & * & $*$ & ns & ns & ns & * & * & $* *$ & * & * & * & \\
\hline
\end{tabular}


Table 5 GWAS results for FEh, TGW, GNS, HD and PH. Information of haplotypes/SNPs physical location, frequency in the collection and effect direction, $P$ values in each environment and the colocated traits (Continued)

\begin{tabular}{|c|c|c|c|c|c|c|c|c|c|c|c|c|c|c|c|c|c|}
\hline \multirow[b]{2}{*}{ Trait } & \multirow[b]{2}{*}{ Haplotype/SNP1 } & \multicolumn{3}{|c|}{ Ref. Seq. v1.0 (Mb) } & \multirow{2}{*}{$\begin{array}{l}\text { Freq. } \\
\text { Effect }^{2}\end{array}$} & \multicolumn{2}{|c|}{ Azul } & \multicolumn{3}{|c|}{ Balcarce } & \multicolumn{6}{|c|}{ Marcos Juárez } & \multirow[b]{2}{*}{ Coloc } \\
\hline & & Chr. & Start & End & & $13^{3}$ & 14 & 13 & 14 & 15 & 11 & 12 & 13 & 14 & 15 & 16 & \\
\hline & Chr5B-B75-Hap4 & $5 B$ & 597.2 & 601.4 & $11-$ & * & ns & ns & ns & ns & * & * & ns & * & $* *$ & ns & \\
\hline & AX-94610041 & $6 \mathrm{~A}$ & 13.8 & - & $46+$ & * & * & $* *$ & * & * & $* *$ & ns & $*$ & * & * & ns & \\
\hline & Chr6A-B43-Hap1 & $6 \mathrm{~A}$ & 591.7 & 592.5 & $61-$ & ns & $* *$ & ns & ns & ns & * & * & $* *$ & * & * & * & \\
\hline & Chr6B-B26-Hар3 & $6 \mathrm{~B}$ & 157.6 & 157.8 & $11+$ & * & $* *$ & ns & ns & ns & ns & ns & ns & * & * & $* *$ & $\mathrm{PH}$ \\
\hline & Chr7A-B24-Hap2 & $7 \mathrm{~A}$ & 68.0 & 70.2 & $8+$ & ns & ns & * & $* *$ & * & * & ns & $* *$ & * & * & ns & \\
\hline \multirow[t]{36}{*}{$\mathrm{PH}$} & Chr1A-B38-Нар3 & $1 \mathrm{~A}$ & 508.6 & 510.4 & $16+$ & * & $* *$ & ns & ns & ns & * & ns & ns & * & ns & ns & \\
\hline & Chr1B-B17-Hap4 & $1 \mathrm{~B}$ & 121.0 & 124.0 & $5+$ & * & * & * & $* *$ & * & $* * *$ & * & $*$ & $*$ & $*$ & $* *$ & \\
\hline & AX-94510167 & $1 \mathrm{~B}$ & 548.7 & - & $21+$ & ns & * & ns & $* *$ & * & $* *$ & ns & ns & * & ns & ns & \\
\hline & Chr1B-B54-Hap1 & $1 \mathrm{~B}$ & 580.5 & 581.2 & $43-$ & * & $* *$ & ns & * & * & * & ns & ns & $* * *$ & $* * *$ & ns & \\
\hline & Lr10 & $1 \mathrm{D}$ & 8.6 & - & $10+$ & $*$ & * & $* *$ & ns & $*$ & ns & $*$ & ns & $*$ & * & ns & \\
\hline & Chr1D-B5-Hap4 & $1 \mathrm{D}$ & 11.6 & 11.9 & $19-$ & * & $* *$ & ns & * & * & * & ns & * & * & $*$ & * & \\
\hline & Chr1D-B10-Hap4 & $1 \mathrm{D}$ & 31.1 & 32.5 & $10+$ & * & ns & $* * *$ & * & ns & * & * & ns & ns & ns & ns & \\
\hline & Chr2A-B39-Hap1 & $2 \mathrm{~A}$ & 611.2 & 611.9 & 70- & $* *$ & ns & $* *$ & ns & ns & * & ns & * & ns & ns & * & \\
\hline & Chr2A-B48-Hар3 & $2 \mathrm{~A}$ & 701.0 & 702.1 & $15-$ & * & *** & * & ns & * & * & ns & ns & * & * & ns & \\
\hline & Chr2B-B56-Hap4 & $2 \mathrm{~B}$ & 469.2 & 476.6 & $6+$ & ns & * & $* *$ & * & ns & * & ns & * & ns & $* *$ & * & \\
\hline & Chr2B-B79-Hар3 & $2 B$ & 664.2 & 664.9 & $5+$ & * & ns & $* *$ & * & ns & $* *$ & ns & * & ns & $* *$ & * & \\
\hline & AX-94687989 & $2 \mathrm{~B}$ & 702.2 & - & $52+$ & ns & $* *$ & ns & ns & ns & ns & * & ns & $* *$ & $*$ & ns & \\
\hline & Chr2B-B97-Hap1 & $2 B$ & 719.5 & 719.5 & $38-$ & ns & $* *$ & ns & ns & ns & ns & * & ns & $* *$ & * & $*$ & \\
\hline & Chr2B-B114-Hap4 & $2 B$ & 762.0 & 763.9 & $8+$ & $* *$ & * & * & ns & * & ns & ns & ns & * & ns & ns & \\
\hline & AX-94585596 & $2 \mathrm{~B}$ & 776.4 & - & $15+$ & * & $* *$ & ns & ns & * & $* *$ & * & * & * & ns & ns & \\
\hline & AX-94624485 & $2 \mathrm{~B}$ & 776.8 & - & $10+$ & * & * & * & ns & ns & $* *$ & * & * & ns & ns & ns & \\
\hline & Chr2B-B122-Нар3 & $2 B$ & 777.1 & 777.2 & $6+$ & * & * & * & ns & * & $* * *$ & $* *$ & $*$ & * & * & ns & \\
\hline & Chr3A-B22-Hap2 & $3 \mathrm{~A}$ & 391.5 & 393.4 & $53+$ & * & * & ns & * & ns & * & $* *$ & * & * & ns & * & \\
\hline & AX-95090222 & $3 \mathrm{~A}$ & 700.6 & - & $18+$ & $* *$ & ns & ns & * & ns & ns & $* *$ & ns & * & ns & ns & \\
\hline & AX-95083205 & $3 A$ & 743.9 & - & $46+$ & $* *$ & * & ns & ns & * & ns & ns & * & * & ns & ns & \\
\hline & Chr3B-B6-Hap1 & $3 B$ & 16.4 & 17.1 & $65-$ & * & * & * & ns & * & ns & * & * & $* *$ & $* *$ & * & \\
\hline & Chr3B-B55-Hap4 & $3 B$ & 511.1 & 513.6 & $6-$ & $* *$ & * & ns & ns & * & ns & ns & ns & ns & * & * & \\
\hline & Chr3B-B60-Hap6 & $3 B$ & 541.5 & 544.5 & $5+$ & * & ns & * & * & ns & $* *$ & * & * & * & $*$ & ns & \\
\hline & Chr3B-B109-Hар2 & $3 B$ & 796.0 & 799.1 & 9- & * & $* *$ & * & ns & ns & ns & ns & ns & ns & * & * & \\
\hline & Chr3B-B111-Hap4 & $3 B$ & 817.4 & 817.8 & $11+$ & $* * *$ & ns & * & * & $* *$ & $* * *$ & * & $* *$ & * & * & * & TGW \\
\hline & Chr4A-B57-Hap6 & $4 \mathrm{~A}$ & 738.7 & 739.7 & 9- & $*$ & ns & ns & $* *$ & ns & $* *$ & ns & ns & ns & $*$ & ns & \\
\hline & Chr4B-B28-Hap5 & $4 \mathrm{~B}$ & 612.2 & 613.2 & $6+$ & * & $* *$ & * & * & * & * & * & * & * & * & ns & \\
\hline & Chr4B-B36-Hap2 & $4 \mathrm{~B}$ & 650.7 & 650.7 & $57-$ & $* *$ & * & * & * & ns & * & $* *$ & $* *$ & ns & * & ns & \\
\hline & Chr5A-B16-Hap4 & $5 A$ & 33.0 & 33.2 & $5+$ & * & * & * & * & * & $* *$ & $* *$ & ns & ns & ns & ns & \\
\hline & Chr5A-B33-Hap4 & $5 \mathrm{~A}$ & 445.2 & 445.2 & 7- & ns & *** & * & ns & ns & * & ns & ns & ns & * & * & FEh \\
\hline & Chr5A-B54-Нар3 & $5 \mathrm{~A}$ & 516.1 & 527.9 & $5+$ & ns & * & * & $* *$ & $* *$ & $* * *$ & ns & ns & * & * & * & \\
\hline & Chr5A-B82-Hap2 & $5 A$ & 611.6 & 613.5 & $10+$ & $*$ & ns & * & ns & ns & * & $* * *$ & ns & * & ns & * & \\
\hline & Chr5B-B26-Hap2 & $5 B$ & 430.7 & 430.7 & $17+$ & $* *$ & * & ns & * & * & * & * & ns & ns & ns & * & \\
\hline & AX-94550178 & $5 B$ & 455.7 & - & $46+$ & ns & ns & $* * *$ & * & * & * & ns & ns & * & ns & ns & \\
\hline & Chr5B-B92-Hap4 & $5 B$ & 683.5 & 684.0 & $5+$ & * & ns & * & * & ns & $* *$ & * & * & * & ns & $* *$ & \\
\hline & Chr6A-B2-Hap5 & $6 \mathrm{~A}$ & 0.6 & 0.8 & $5+$ & * & ns & $* *$ & $* *$ & * & ns & ns & ns & * & * & ns & \\
\hline
\end{tabular}


Table 5 GWAS results for FEh, TGW, GNS, HD and PH. Information of haplotypes/SNPs physical location, frequency in the collection and effect direction, $P$ values in each environment and the colocated traits (Continued)

\begin{tabular}{|c|c|c|c|c|c|c|c|c|c|c|c|c|c|c|c|c|c|}
\hline \multirow[b]{2}{*}{ Trait } & \multirow[b]{2}{*}{ Haplotype/SNP ${ }^{1}$} & \multicolumn{3}{|c|}{ Ref. Seq. v1.0 (Mb) } & \multirow{2}{*}{$\begin{array}{l}\text { Freq. } \\
\text { Effect }^{2}\end{array}$} & \multicolumn{2}{|c|}{ Azul } & \multicolumn{3}{|c|}{ Balcarce } & \multicolumn{6}{|c|}{ Marcos Juárez } & \multirow[b]{2}{*}{ Coloc } \\
\hline & & Chr. & Start & End & & $13^{3}$ & 14 & 13 & 14 & 15 & 11 & 12 & 13 & 14 & 15 & 16 & \\
\hline & Chr6A-B4-Hap2 & $6 \mathrm{~A}$ & 2.2 & 2.7 & $26+$ & $*$ & ns & * & * & $* *$ & ns & * & ns & ns & * & ns & \\
\hline & Chr6A-B4-Hap3 & $6 \mathrm{~A}$ & 2.2 & 2.7 & $17-$ & $* *$ & * & $*$ & ns & * & ns & * & ns & * & $* *$ & * & \\
\hline & Chr6A-B16-Hap1 & $6 \mathrm{~A}$ & 48.6 & 49.8 & $57+$ & ns & ns & ns & $*$ & ns & * & * & ns & $* *$ & * & $* *$ & \\
\hline & Chr6A-B17-Hар3 & $6 \mathrm{~A}$ & 50.3 & 51.4 & 33- & ns & * & $*$ & * & ns & $* *$ & ns & * & * & ** & ns & \\
\hline & Chr6A-B54-Hap5 & $6 \mathrm{~A}$ & 610.0 & 610.2 & $6+$ & ns & ns & * & $*$ & ns & $* * *$ & ns & $* *$ & ns & ns & ns & \\
\hline & Chr6B-B7-Hap2 & $6 \mathrm{~B}$ & 19.8 & 20.7 & $82-$ & $* *$ & $* * *$ & $* *$ & * & ns & * & $* * *$ & * & * & * & $*$ & \\
\hline & Chr6B-B20-Hap4 & $6 B$ & 124.4 & 126.1 & $6+$ & * & ns & ns & ns & ns & $* *$ & * & * & ns & * & * & \\
\hline & Chr6B-B25-Hap4 & $6 B$ & 151.4 & 156.4 & $25-$ & $*$ & $* *$ & $*$ & * & $* *$ & ns & ns & ns & * & * & ns & \\
\hline & Chr6B-B26-Нар3 & $6 B$ & 157.6 & 157.8 & $11-$ & $*$ & $* * *$ & ns & * & ns & * & * & * & ns & * & $* * *$ & $\mathrm{HD}$ \\
\hline & Chr6B-B31-Hap2 & $6 B$ & 195.3 & 197.8 & $5+$ & $*$ & ns & $* *$ & $* *$ & * & $* *$ & ns & ns & * & * & * & \\
\hline & AX-94943227 & $6 \mathrm{~B}$ & 234.8 & - & $17+$ & $* *$ & $* * *$ & $* *$ & * & * & * & $* * *$ & * & $*$ & * & $*$ & \\
\hline & Chr6B-B47-Hap3 & $6 B$ & 480.4 & 491.6 & $24-$ & ns & ns & ns & * & $* *$ & ns & ns & ns & * & ns & * & \\
\hline & AX-94476474 & $7 \mathrm{~A}$ & 561.6 & - & $14+$ & $* *$ & * & $*$ & ns & ns & ns & ns & $* *$ & $*$ & * & ns & \\
\hline & Chr7B-B14-Hap4 & $7 \mathrm{~B}$ & 59.6 & 61.6 & $22-$ & $* * *$ & ns & ns & ns & * & ns & * & ns & ns & * & ns & \\
\hline & Chr7B-B76-Hap3 & $7 \mathrm{~B}$ & 743.5 & 743.6 & $10-$ & $* *$ & $* * *$ & * & * & ns & * & ns & * & $* *$ & $* *$ & * & \\
\hline
\end{tabular}

${ }^{1}$ Information about SNPS ID and/or gene molecular marker that were involved in the haplotypes was detailed in the Additional file 6: Table S5. ${ }^{2}$ Freq. $=$ Number of genotypes that present the SNP / haplotype in the collection. Effect (+) the SNP / haplotype increases the phenotypic value and (-) reduces the phenotypic value. ${ }^{3}$ Years after 2000. Only SNPs that passed selection criteria in GWAS are presented in the table. ns = not significant, ${ }^{*} P<0.05,{ }^{* *} P<0.001,{ }^{* * *} P<0.0001, "-"=$ not available

six haplotypes/markers showed significant associations across all locations, including haplotype Chr1B-B17-Hap4 and SNP AX-94943227 on chromosome 6B that showed consistent significant effects in all tested locations and years. Three haplotypes significantly associated with $\mathrm{PH}$ colocated with traits FEh, TGW and HD, with contributions previously described (Table 5).

\section{Discussion}

\section{Genetic structure and haplotype characterization}

In this work, results of a model-based cluster analysis differentiated four subpopulations, whereas previously [32], using the same 102 cultivars and a small number of markers associated with genes of agronomic interest and neutral markers (SSRs and ISBPs), detected three subpopulations, which nonetheless and as expected overlapped with the four identified here. In general terms, subpopulations 1 and 2 in our study matched with subpopulations 1 and 3 of the previous work, in including introductions of European origin and traditional germplasm, respectively. Subpopulations 3 and 4 in our study matched subpopulation 2 of the previous work, all including in most cases germplasm with pedigrees of CIMMYT origin. Differences between subpopulations 3 and 4 may be related to the presence of the $1 \mathrm{BL} / 1 \mathrm{RS}$ wheat-rye translocation, as $24 / 26$ cultivars in subpopulation 3 carry this, whereas no cultivar is in a similar situation in subpopulation 4 (data not shown). This translocation has been widely used in breeding to achieve resistance to several pathogens and insects, to broaden adaptation and to increase yield [34]. Argentina is no exception, as modern cultivars carrying 1BL/1RS have shown: Klein Gladiador, Klein Nutria, Klein Yarará, LE 2333, LE 2341 and ACA 906, released between 2009 and 2010, confirm the consistent contribution of the translocation to biotic and abiotic stress tolerance. In spite of these advantages, special attention should be given to the use of $1 \mathrm{BL} / 1 \mathrm{RS}$, due to its detrimental effects on gluten strength and bread-making quality, albeit that they vary depending upon the genetic background [35].

Wheat has been exposed to intense artificial and natural selection since its domestication, resulting in large HBs as observed in elite germplasm [18, 36]. This issue can be observed in our work with the just mentioned presence of the $1 \mathrm{BL} / 1 \mathrm{RS}$ wheat-rye translocation (Fig. 1b). In any case, we must also take into account the position of the $\mathrm{HB}$ in the chromosome, as we see in Fig. 1a, where large haplotypes $(>30 \mathrm{Mb})$ are formed in centromeric and pericentromeric regions of each chromosome. As previously reported in the work of [37], these regions can be a challenge when introducing allelic variants of interest in the region and reducing the size of the HBs.

\section{GWAS analysis}

Yield potential related traits:

Fruiting efficiency has been proposed as a promising trait for increasing yield potential in wheat $[12,13,28,38$, 

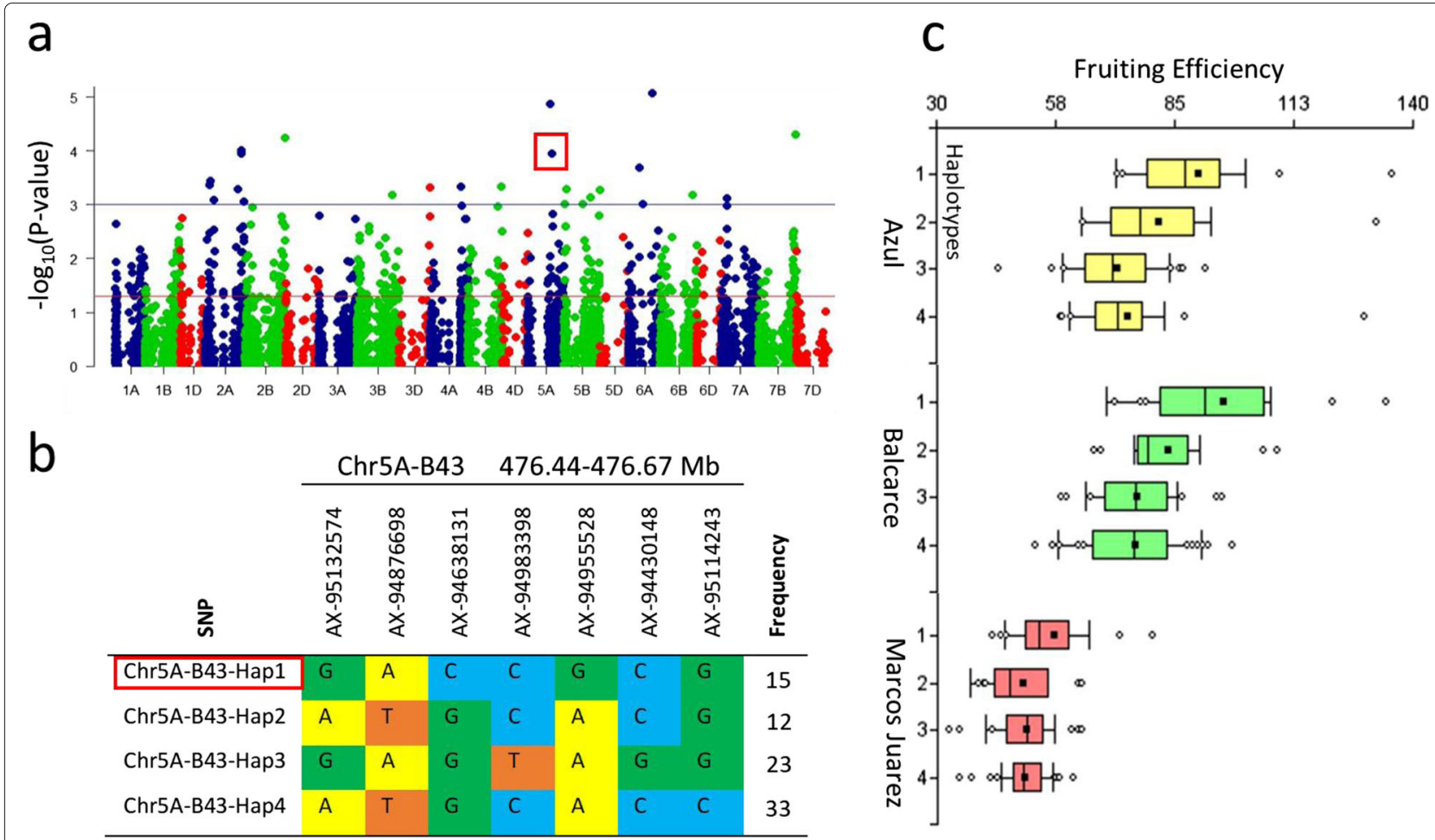

Fig. 2 a Manhattan plot on 21 wheat chromosomes for fruiting efficiency at harvest (FEh) in Balcarce 2013. Red line represents the GWAS threshold of $P<0.05=-\log _{10}(P$-value $)=1.3$ and blue line represents the GWAS threshold of $P<0.001=-\log _{10}(P$-value $)=3$. Red square highlights the Chr5A-B43-Hap1 associated with FEh in five of ten tested environments. b Haplotype block based on seven SNP markers located between 476.44 and 476.67Mb on chromosome 5A, named Chr5A-B43. Four different haplotype variants (Hap1-Hap4) are present at different frequencies in the analyzed population. Red rectangle highlights the Chr5A-B43-Hap1 associated with FEh. c Boxplots indicate the phenotype values corresponding to the four different haplotype groups in the three evaluated locations. Hap1 was associated significantly high FEh in all locations

39]. We detected 15 significant haplotypes/markers with positive effects on FEh and only two negatives (Table 5). The strongest genetic associations for FEh were detected in the Southern locations Azul and Balcarce, with lower associations observed in Marcos Juárez. These results imply FEh could provide an avenue to increase yield in regions where yield potential is high, as in the case of the Southern locations in our study.

In a previous study, a positive correlation between FEh and grain number was detected [13, 39]. Here, we detected a haplotype Chr2A-B49-Hap2 (704.8-705.8Mb) on chromosome $2 \mathrm{~A}$, that positively modifies both the traits FEh and GNS (Table 5). In a further study, [40] detected a SNP associated with FE and grains per spikelet (RFL_Contig3780_64), located at $676.24 \mathrm{Mb}$ on chromosome 2A. They proposed CONSTANS $4(\mathrm{CO} 4)$ and TaVrs1 genes as candidates to explain the variation detected. However, based upon the IWGSC Ref Seq. V1.0, CO4 is located at $594.58 \mathrm{Mb}(81.66 \mathrm{Mb}$ proximal to RFL_Contig3780_64). Our haplotype Chr2A-B49-Hap2 is located 28.56 Mb distal from RFL_Contig3780_64. The physical distance between the Chr2A-B49-Hap2 and $\mathrm{CO} 4$ is estimated as $110.22 \mathrm{Mb}$, and, taking into account the critical value for the decay of linkage desequilibrium (LD) by 0.1 is estimated as an average of $50 \mathrm{Mb}$ for the wheat genome [41], would discard $\mathrm{CO} 4$ as a candidate for the FEh QTL detected at Chr2AB49-Hap2. On the other hand, the gene TaVrs1, also called GNI-A1, was recently cloned and characterized by [42]; unfortunately the GNI-A1 gene is not assembled on IWGSC Ref Seq. V1.0 chromosome 2A and its location cannot be compared to the Chr2A-B49-Hap2 haplotype.

In contrast, a negative relationship between FEh and TGW has also been described [28, 39]. In the current work, we detected haplotype Chr6A-B24-Hap2 (205.1233.3Mb) that increases FEh values, but penalizes TGW.

Little is known about the relationship between FEh and $\mathrm{PH}$. We detected a haplotype on chromosome 5A, Chr5AB33-Hap4 (445.2 Mb) that associates with both traits, but in opposite directions.

Another relevant trait in the determination of yield potential is grain weight. We detected six significant haplotypes/markers with positive effects and five with negative effects on TGW (Table 5). This list includes four haplotypes on chromosome $6 \mathrm{~A}$ associated with TGW, one of them Chr6A-B24-Hap2 (205.1-233.3Mb). This haplotype, negatively associated with TGW and positively associated 
with FEh, was located $4.5 \mathrm{Mb}$ proximal to the gene TaGW2-A1, which is located at $237.75 \mathrm{Mb}$ on chromosome $6 \mathrm{~A}$ and has been shown to be related to grain weight [43]. Non-functional mutants for this gene increase the weight and size of wheat grains [44]. Shared function and physical location would turn TaGW2-A1 into a promising candidate gene for Chr6A-B24-Hap2. On the other hand, the gene TaGS5-3A located at $176.55 \mathrm{Mb}$ on chromosome $3 \mathrm{~A}$ has also been associated with larger grain size and higher thousand grain weight [45]. We found one haplotype and one SNP associated with TGW on chromosome 3A (Table 5), but they were located too far away from TaGS5-3A (123 and 510Mb apart, respectively) to be considered as a gene candidate. In the opposite direction to that observed for FEh, the strongest genetic associations for TGW were detected in the Northern location Marcos Juárez, promoting TGW as an interesting trait to increase yield for those latitudes.

For GNS, [46] used GWAS to detect a region located at $691.22 \mathrm{Mb}$ on chromosome $2 \mathrm{~A}$ that significantly affects the number of grains per spike. We detected the haplotype Chr2A-B49-Hap2, located at 704.8-705.8 Mb, that positively affects GNS, only $13 \mathrm{Mb}$ distal from the [46] region.

\section{Crop adaptation related traits:}

For HD, we found that the Ppd-D1 gene marker [47] is the strongest associated with the trait, especially in the location Marcos Juárez, situated at the lowest latitude in our study. These results agree with those obtained by [33], where the $P p d-D 1$ gene was found to be the main determinant of life cycle in Argentinean wheat cultivars.

On chromosome 1B, we detected three haplotypes associated with HD. [48] proposed the gene TaFT-B3 $(581.4 \mathrm{Mb})$ as a HD modifier for short days on this chromosome. Our nearest significant haplotype, Chr1B-B76Hap2 (Table 5), is located $61.7 \mathrm{Mb}$ distal from TaFT-B3, being slightly farther than the critical value of 0.1 for $L D$ decay estimated as $50 \mathrm{Mb}$ [41].

On the other hand, [49] showed that loss of function mutants of the PHYTOCLOCK 1 (WPCL1) gene, located at $740.1 \mathrm{Mb}$ on chromosome $3 \mathrm{~A}$, were associated with extra-early flowering time. We detected four haplotypes on chromosome 3A associated with HD. Our nearest significant haplotype, Chr3A-B-Hap4 (Table 5), is located $39.5 \mathrm{Mb}$ proximal to $W P C L 1$ positioning WPCL1 as candidate for the Chr3A-B-Hap4 HD association.

Finally, no significant associations were detected for HD with the genes Vrn-A1 (5A) [50], Vrn-B1 (5B), Vrn-D1 (5D) [51] and Ppd-B1 (2B) [52], indicating that this set of genes did not appear to affect heading time in our cultivar collection. The most likely explanation is that may come from the field conditions where the collection was evaluated, with satisfied vernalization requirements, or potentially high epistatic interactions among vernalization genes, causing difficulties in the detection of minor effects on flowering time.

In the case of $\mathrm{PH}$, we did not find significant associations between the trait and the molecular markers for the "green revolution" dwarfism genes $R h t-B 1$ and Rht$D 1$, described by [53]. Although the collection presented a wide variation in $\mathrm{PH}$, it should be mentioned that the wheat collection used in our study is mainly composed of semi-dwarf elite germplasm and that the Rht-B1 and Rht-D1 genes are balanced.

Within the 51 significant associations detected, the chromosome 1B haplotype Chr1B-B17-Hap4 and the chromosome 6B SNP AX-94943227 (234.8 Mb) were particularly noteworthy, since they were significant across the three locations in all analyzed years. To our knowledge, there are no plant height related genes described on these chromosomes, which suggest these consistent $\mathrm{PH}-\mathrm{HB} / \mathrm{SNP}$ associations are promising targets for further gene cloning projects.

We detected four haplotypes on chromosome 5A associated with PH. In a mapping work [54] located the GAresponsive Rht genes $R h t 9$ and $R h t 12$ on chromosome arm 5AL, where Rht9 was linked to the SSR barc151 (558.34 $\mathrm{Mb})$ and $R h t 12$ was located $5.4 \mathrm{cM}$ from the SSR Xgwm291 (698.19 Mb). Our haplotype Chr5A-B54-Hap3 mapped at 516.1-527.9 Mb might be associated with Rht9 linked to SSR barc151 at $558.34 \mathrm{Mb}$. However, such an association with Rht12 has to be discarded in our study, since additional $\mathrm{PH}$ haplotypes are placed in genetic regions distant to this $\mathrm{PH}$ gene.

On chromosome 6A, we detected six haplotypes associated with $\mathrm{PH}$; in particular, the haplotype Chr6A-B54Hap5 $(610.0 \mathrm{Mb})$ was significantly associated with $\mathrm{PH}$ in the three locations and in all evaluation years, except Balcarce 2015. However, none of the associated haplotypes was located in physical positions close to any of the known $\mathrm{PH}$ genes on 6A, namely Rht25 (144.0-148.3 Mb) detected by [55] and Rht18/Rht14/Rht24(413.73 Mb) described previously [56].

It is important to highlight that small association panels have been shown to increase both type 1 and type 2 error rates, failing to detect true associations while also generating a higher rate of false positive associations [57]. In this work, we used a small association panel (102 cultivars), but we used a conservative approach for GWAS, in order to reduce spurious associations. However, we may be failing to detect genomic regions that have low rates of explanation of phenotypic values or are found in low frequency in the panel. It is recommended to validate the associations reported in independent populations before being used in wheat breeding programs. 


\section{Conclusion}

The genetic structure of the Argentinean hexaploid wheat collection was determined by the use of SNP markers, in which a strong relationship was displayed between the subpopulations obtained and the germplasm origin used by the main breeding programs in the country. Based on SNP and gene-related markers physically anchored to the IWGSC Ref Seq v1.0, a haplotype map was constructed, allowing the detection of highly conserved and selected regions like the $1 \mathrm{BL} / 1 \mathrm{RS}$ translocation. A GWAS detected ninety-seven chromosome region associated with yield components and adaptation. In the case of yield components, we highlight regions on chromosomes $1 \mathrm{~A}, 2 \mathrm{~A}, 3 \mathrm{~B}$, $4 \mathrm{~A}, 5 \mathrm{~A}, 6 \mathrm{~A}$ and $7 \mathrm{~A}$ associated with FEh, particularly at higher yield potential locations. For adaptation, the most relevant effect on HD was the Ppd-D1 gene on chromosome $2 \mathrm{D}$, being the main determinant in the variations in the life cycle of the Argentinean wheats. The use of the IWGSC Ref Seq v1.0 allowed us to precisely compare all detected associated regions with genes and QTLs reported in previous studies.

\section{Methods}

Plant material

A previously described [32] set of 102 bread wheat cultivars that includes old (such as cv. Barletta 77 released in 1927) and recent (up to 2010) commercial cultivars were selected from the main wheat breeding companies in Argentina and used for the haplotype block construction and GWAS analysis. Seed stocks were kindly provided by the Instituto Nacional de Tecnología Agropecuaria (INTA) Experimental Station Marcos Juárez, Wheat Germplasm Bank (Marcos Juárez, Argentina).

\section{Genotypic data}

The population was genotyped at the Instituto de Genética Veterinaria (IGEVET) Genotyping Laboratory, La Plata, Argentina, using the 35k Axiom Wheat Breeder's Genotyping Array [22]. Information about the 35k SNPs Axiom array is publicly available in CerealsDB ${ }^{1}$. SNPs with minor allele frequency (MAF) $<0.10$ and/or with more than $10 \%$ of missing data were discarded. The polymorphic SNPs were genetically anchored to specific wheat chromosomes according to previously described map information [22]. In a second step, linkage information from three biparental mapping populations was used to anchor new markers to wheat chromosomes. Additionally, several SNPs were anchored by transforming the SNP nucleotide information into numerical format and using this information as a phenotypic value for an association study. SNPs with an association of $P \leq 0.0001$ to a chromosome were assigned to it (Additional file 1:

\footnotetext{
${ }^{1}$ http://www.cerealsdb.uk.net/cerealgenomics/CerealsDB/indexNEW.php
}

Table S1). The physical position of each SNP marker in each wheat chromosome was determined by blasting their flanking sequences against the IWGSC Ref Seq v1.0 genome assembly [21]. Molecular data of markers associated with agronomic traits previously described [32] were also included. In the case of adaptation traits, markers were included for the genes Rht-B1, Rht-D1 [53], Vrn-A1 [50], Vrn-B1, Vrn-D1 [51], Elf3-D1 [58], $P p d-B 1$ [52] and Ppd-D1 [47]. Regarding diseases, markers for leaf rust resistance genes $\operatorname{Lr} 10$ [59], Lr34 [60] and $\operatorname{Lr24}$ [61] were incorporated. Additionally, industrial quality related markers, Ppo-A1, Ppo-D1 [62], $R$ $A 1, R-B 1, R-D 1$ [63], Glu-B1al [64], Wx-A1,Wx-B1 [65], Vp1-B3 [66], Glu-A3 [67], PinA-D1 and PinB-D1 [68] were included. Finally, high-molecular-weight glutenin subunit (HMW-GS) loci Glu-A1, Glu-B1 and Glu-D1 were characterized based upon the protocol described by [69].

\section{SNP-based haplotype construction}

The SNP-based haplotype structure for each wheat chromosome was evaluated using the Haploview 4.2 software package [70]. The package defines haplotype blocks (HB) and provides the number of haplotypes and their physical length (bp) for each block, as well as the number of tagged SNPs based on the solid spine of linkage disequilibrium (LD) (Extend spine if $\mathrm{D}^{\prime}>0.8$ ). This means that the first and the last marker in a block are in strong $\mathrm{LD}$ with the intermediate markers that are not necessarily in LD with each other [18, 70]. The HBs from Haploview were converted to a table by using an in-house python script available on GitHub ${ }^{2}$. The script transforms the table file results into a suitable input for the 'Genome Association and Prediction Integrated Tool' (GAPIT) software [71]. Detailed information about the haplotype map constructed is given in Additional file 4: Table S3.

\section{Field experiments and phenotypic trait assessment}

In Marcos Juárez, the field experiments were carried out during 2011 to 2016 (6 years) at INTA Experimental Station Marcos Juárez ( $32^{\circ} 42^{\prime} \mathrm{S}, 62^{\circ} 07^{\prime} \mathrm{W}, 114$ m.a.s.l.). In Azul, the field experiments were carried out during 2013 and 2014 at the Experimental Field of the Faculty of Agronomy, Universidad Nacional del Centro de la Provincia de Buenos Aires, Azul (36 48 $\mathrm{S}$; $59^{\circ} 51^{\prime}$ W, $137 \mathrm{~m}$ a.s.l.). In Balcarce, the field experiments were carried out during crop seasons 2013, 2014 and 2015 at INTA Experimental Station Balcarce $\left(37^{\circ} 45^{\prime} \mathrm{S}\right.$; $55^{\circ}$ $18^{\prime} \mathrm{W} ; 130 \mathrm{~m}$ a.s.l.). In all experiments except Marcos Juárez 2011 and Balcarce 2013 a Randomized Complete Block Design (RCBD) with two replications was used.

\footnotetext{
${ }^{2}$ https://github.com/INTABiotechMJ/haploview2gapit
} 
Table 6 Description of experiments for the Argentinean hexaploid wheat association mapping collection

\begin{tabular}{|c|c|c|c|c|c|}
\hline Exp. & Location & Year & Blocks & Exp. unit & Traits phenotyped \\
\hline 1 & $\mathrm{Azul}^{1}$ & 2013 & 2 & One meter row & FEh, TGW, GNS, HD, PH \\
\hline 2 & Azul & 2014 & 2 & One meter row & FEh, TGW, GNS, HD, PH \\
\hline 3 & Balcarce $^{2}$ & $2013^{4}$ & 1 & Half meter row & FEh, TGW, GNS, HD, PH \\
\hline 4 & Balcarce & 2014 & 2 & $\operatorname{Plot}^{5}$ & FEh, TGW, GNS, HD, PH \\
\hline 5 & Balcarce & 2015 & 2 & Plot & FEh, TGW, GNS, HD, PH \\
\hline 6 & Marcos Juárez ${ }^{3}$ & 2011 & 1 & One meter row & $\mathrm{HD}, \mathrm{PH}$ \\
\hline 7 & Marcos Juárez & 2012 & 2 & One meter row & FEh, TGW, GNS, HD, PH \\
\hline 8 & Marcos Juárez & 2013 & 2 & One meter row & FEh, TGW, GNS, HD, PH \\
\hline 9 & Marcos Juárez & 2014 & 2 & Plot & FEh, TGW, GNS, HD, PH \\
\hline 10 & Marcos Juárez & 2015 & 2 & Plot & FEh, TGW, GNS, HD, PH \\
\hline 11 & Marcos Juárez & 2016 & 2 & One meter row & FEh, TGW, GNS, HD, PH \\
\hline
\end{tabular}

${ }^{1}$ Conducted under no nutrient limitations and rainfed conditions with chemical control of pests and fungal diseases. ${ }^{2}$ Conducted under no nutrient or water limitations, with chemical control of pests and fungal diseases. ${ }^{3}$ Conducted under rainfed conditions without disease control. ${ }^{4}$ Five sowing dates were used for all 102 cultivars; heading date information was collected for each cultivar at each sowing date and used for grouping cultivars into three groups of similar heading date. In 2014 and 2015 experiments at Balcarce, each cultivar was sown at one of each three sowing dates, in order for all cultivars to have similar heading date (around the first week of November). ${ }^{5}$ Plot: $5 \mathrm{~m}$

long seven-row plot, with a $0.2 \mathrm{~m}$ inter-row distance

More detailed information of the field experiments carried out for GWAS is described in Table 6. The collection was evaluated for two crop adaptation traits: heading date (HD) and plant height (PH), and three yield component related traits: fruiting efficiency at harvest (aka spike fertility index; FEh), grain number per spike (GNS) and thousand grain weight (TGW). The HD (in days) was measured from emergence until $50 \%$ of the spike had emerged from the flag leaf [72]. The $\mathrm{PH}$ (in $\mathrm{cm}$ ) was determined after maturity as the mean of 10 to 20 randomly selected plants (according to experiment) by measuring the main tiller of each plant from the ground to the top of the spike, excluding awns. At maturity, 5 to 15 random spikes (according to experiment), were sampled. They were cut at the lowest spikelet level, weighed and threshed. Spike chaff dry weight (in g) was calculated as the difference between total spike dry weight before threshing and total grain weight. The FEh (in grains g-1) was calculated at harvest as the quotient between grain number and spike chaff dry weight per sample according to [26]. The GNS was measured as the mean of the total number of grains in the selected spikes. The TGW (in g) was determined by weighing 1000 grains. In MsJz, the phenotypic data was collected using Phenobook software [73]. All the phenotypic data used for GWAS is given in Additional file 5: Table S4.

\section{Statistical analysis}

The best linear unbiased predictors (BLUPs) of traits in each environment were obtained (except for Marcos Juárez 2011 and Balcarce 2013, where no experimental replicates were made) and were used for Pearson's correlation analyses and broad sense heritability estimates.
Broad sense heritability $\left(H^{2}\right)$ was estimated using the formula

$$
H^{2}=\sigma_{G}^{2} /\left(\sigma_{G}^{2}+\left(\sigma_{e}^{2} / r\right)\right)
$$

where $\sigma_{G}^{2}$ is the genotypic variance, $\sigma_{e}^{2}$ is the residual variance, and $r$ is the number of environments. The genotype by environment variance was used as error variance $\left(\sigma_{e}^{2}=\sigma_{G x E}^{2}\right)$.

\section{Haplotype-based GWAS}

GWAS were performed using 4516 haplotypes (from 1268 $\mathrm{HB}$ ) and 495 informative SNP/gene markers anchored to the wheat genome and the SUPER method [74] implemented in the R package GAPIT [71]. In order to reduce spurious associations, genetic structure in the population level ( $Q$ values) was evaluated using R STRUCTURE software [31].The R scripts used for GWAS are available on GitHub ${ }^{3}$ From the GWAS for each trait, we selected haplotypes/markers that were significant $(P<0.05$, markerwise) at four environments, with at least one environment with highly significant differences $(P<0.001)$. All the haplotypes/markers that satisfied these criteria are presented in Table 5. Based on the formula described in [75], the probability of a haplotype/marker being significant by chance simultaneously for four GWAS was estimated to be less than $1.25 \mathrm{E}-07(0.05 \times 0.05 \times 0.05 \times 0.001)$. Based on this number, the probability of at least one error in the 5011 haplotypes/SNPs was estimated as 1 - (1 $1.25 \mathrm{E}-07)^{5011}=0.00063$ (per trait).

\footnotetext{
${ }^{3}$ https://github.com/INTABiotechMJ/AssociationMappingScripts
} 


\section{Supplementary information}

Supplementary information accompanies this paper at https://doi.org/10.1186/s12870-019-2015-4.

Additional file 1: Table S1. Polymorphic SNPs and gene-related molecular markers genetically and physically anchored to IWGSC Ref Seq v1.0. based on 102 Argentinean hexaploid wheats.

Additional file 2: Table S2. STRUCTURE Q matrix of the 102 Argentinean hexaploid wheats cultivars.

Additional file 3: Figure S1. Graphical representation of STRUCTURE Q matrix in the 102 Argentinean hexaploid wheats cultivars.

Additional file 4: Table S3. Haplotype-based map used for HB characterization and GWAS in the Argentinean hexaploid wheat collection.

Additional file 5: Table S4. Phenotypic data from Balcarce (2013-2015), Azul (2013-2014) and Marcos Juárez (2011-2016) for FEh, GNS, TGW, HD and PH used in GWAS.

Additional file 6: Table S5. Detailed information of the significant associated Haplotypes/markes detected by GWAS for FEh, TGW, GNS, PH and HD using the 102 Argetninean hexaploid wheat collection including all the SNPs/markers ID.

\section{Abbreviations}

$\sigma_{e}^{2}$ : Error variance; $\sigma_{e}^{2}$ : Residual variance; $\sigma_{G}^{2}$ : Genotypic variance; $\sigma_{G \times E}^{2}$ : Genotype by environment variance; bp: Base pair; BLUP: The best linear unbiased predictors; CIMMYT: Centro Internacional de Mejoramiento de Maiz y Trigo; FEh: Fruiting efficiency at harvest; GNS: Grain number per spike; GWAS: Genome wide association study; $H^{2}$ : Broad sense heritability; HB: Haplotype block; HD: Heading date; INTA: Instituto Nacional de Tecnologia Agropecuaria; ISBP: Insertion site-based polymorphism; IWGSC: International wheat genome sequencing consortium; LD: Linkage disequilibrium; MAF: Minor allele frequency; Mb: Mega base; PH: Plant height; r: Number of environments; RCBD: Randomized complete block design; SNP: Single nucleotide polymorphism; SSR: Simple sequence repeat; TGW: Thousand grain weight

\section{Acknowledgements}

The authors wish to thank to the International Wheat Genome Sequencing Consortium (IWGSC) for pre-publication access to the IWGSC RefSeq v1.0. SMLB and JMC were research fellows of the National Council for Science and Technology (CONICET), Argentina. IAR was supported by a grant from Monsanto's Beachell-Borlaug Scholarship Program. The authors thank Viviana Echenique and Gabriela Tranquilli, leaders of Project PICT-2012-0660 for the financial support of genotyping. The authors acknowledge the technical assistance of Romina Aurelia, Maria Donato, Lorena Cortizo, Mauro Martinez and Horacio Dalla Valle.

\section{Authors' contributions}

$S M L B, I A R, P A, M H, W J R, A P$ and $L V$ designed the research. SMLB, IAR, JC, LV and WJR carried out the experiments. SMLB, IAR, MD, and LV collected phenotypic data. JC developed the computer programs. SMLB, LV, MBC and $J C$ performed the data analysis. SMLB, LV and JC drafted the manuscript. WJR, $\mathrm{PA}, \mathrm{MH}$, and AP were involved in improving the manuscript. All the authors critically read and approved the manuscript.

\section{Funding}

This work was supported by the following projects: PNBIO1131042 from the National Institute of Agricultural Technology (INTA), Argentina; FONCYT PICT-2012-0660; CONICET PIP 11420090100291; UNICEN 03/A192; UNICEN 03/A215; and CIC-PBA N ${ }^{\circ} 673 / 06$. IAR was supported by a grant from Monsanto's Beachell-Borlaug Scholarship514 Program.

\section{Availability of data and materials}

The datasets supporting the conclusions of this article are included within the article.

\section{Ethics approval and consent to participate}

Not applicable.

\section{Consent for publication}

Not applicable.

\section{Competing interests}

IAR was supported by a grant from Monsanto's Beachell-Borlaug Scholarship Program. The authors declare that they have no competing interests.

\section{Author details}

${ }^{1}$ Laboratorio de Biología Funcional y Biotecnología (BIOLAB)-INBIOTECCONICET, Facultad de Agronomía, UNCPBA., Av. República de Italia, 7300 Azul, Argentina. ${ }^{2}$ Unidad Integrada Balcarce Facultad de Ciencias Agrarias, Universidad Nacional de Mar del Plata - Estación Experimental Agropecuaria Balcarce, Instituto Nacional de Tecnología, Ruta 226, km 73.5, 24105 Balcarce, Argentina. ${ }^{3}$ Laboratorio de Biotecnología, EEA INTA Marcos Juárez, Grupo Biotecnología y Recursos Genéticos, Instituto Nacional de Tecnología Agropecuaria, Ruta 12 s/n, 2580 Marcos Juárez, Argentina. ${ }^{4}$ Consejo Nacional de Investigaciones Científicas y Técnicas (CONICET)., Buenos Aires, Argentina.

Received: 9 April 2019 Accepted: 3 September 2019

Published online: 16 December 2019

\section{References}

1. Ramankutty N, Mehrabi Z, Waha K, Jarvis L, Kremen C, Herrero M, Rieseberg LH. Trends in global agricultural land use: implications for environmental health and food security. Annu Rev Plant Biol. 2018;69: 789-815.

2. Reynolds M, Foulkes MJ, Slafer GA, Berry P, Parry MA, Snape JW, Angus WJ. Raising yield potential in wheat. J Exp Bot. 2009;60(7):1899-918.

3. Hall AJ, Richards RA. Prognosis for genetic improvement of yield potential and water-limited yield of major grain crops. Field Crops Res. 2013;143:18-33

4. Zhao C, Liu B, Piao S, Wang X, Lobell DB, Huang Y, Huang M, Yao Y, Bassu S, Ciais $P$, et al. Temperature increase reduces global yields of major crops in four independent estimates. Proc Natl Acad Sci. 2017;114(35): 9326-31.

5. García GA, Miralles DJ, Serrago RA, Alzueta I, Huth N, Dreccer MF. Warm nights in the argentine pampas: Modelling its impact on wheat and barley shows yield reductions. Agric Syst. 2018;162:259-68.

6. Foulkes M, Reynolds M. Breeding challenge: improving yield potential. In: Crop Physiology (Second Edition). Elsevier; 2015. p. 397-421. https://doi. org/10.1016/b978-0-12-417104-6.00016-9.

7. Flohr B, Hunt J, Kirkegaard J, Evans J, Swan A, Rheinheimer B. Genetic gains in nsw wheat cultivars from 1901 to 2014 as revealed from synchronous flowering during the optimum period. Eur J Agron. 2018;98: $1-13$.

8. Peltonen-Sainio P, Jauhiainen L, Laurila IP. Cereal yield trends in northern european conditions: Changes in yield potential and its realisation. Field Crops Res. 2009;110(1):85-90.

9. Lopes M, Reynolds M, Manes Y, Singh R, Crossa J, Braun H. Genetic yield gains and changes in associated traits of cimmyt spring bread wheat in a "historic" set representing 30 years of breeding. Crop Sci. 2012;52(3): 1123-31.

10. François $O$. Running structure-like population genetic analyses with $r . R$ Tutor Popul Genet U Grenoble-Alpes. 20161-9. http://membrestimc. imag.fr/Olivier.Francois/tutoRstructure.pdf. Accessed 13 Sept 2019.

11. Lo Valvo PJ, Miralles DJ, Serrago RA. Genetic progress in argentine bread wheat varieties released between 1918 and 2011: Changes in physiological and numerical yield components. Field Crops Res. 2018;221: 314-21.

12. Alonso M, Mirabella N, Panelo J, Cendoya M, Pontaroli A. Selection for high spike fertility index increases genetic progress in grain yield and stability in bread wheat. Euphytica. 2018;214(7):112.

13. Abbate $P$, Andrade F, Lazaro L, Bariffi J, Berardocco H, Inza V, Marturano F. Grain yield increase in recent argentine wheat cultivars. Crop Sci. 1998;38(5):1203-9.

14. Holland J. Genetic architecture of complex traits in plants. Curr Opin Plant Biol. 2007;10(2):156-61. https://doi.org/10.1016/j.pbi.2007.01.003.

15. Huang $X$, Han B. Natural variations and genome-wide association studies in crop plants. Ann Rev Plant Biol. 2014;65:531-51.

16. Ganal MW, Altmann T, Röder MS. Snp identification in crop plants. Curr Opin Plant Biol. 2009;12(2):211-7.

17. Tian F, Bradbury PJ, Brown PJ, Hung H, Sun Q, Flint-Garcia S, Rocheford TR, McMullen MD, Holland JB, Buckler ES. Genome-wide association study of leaf architecture in the maize nested association mapping 
population. Nat Genet. 2011;43(2):159-62. https://doi.org/10.1038/ng 746.

18. Hao C, Wang Y, Chao S, LiT, Liu H, Wang L, Zhang X. The iselect $9 \mathrm{ksnp}$ analysis revealed polyploidization induced revolutionary changes and intense human selection causing strong haplotype blocks in wheat. Sci Rep. 2017;7:41247.

19. Korte A, Farlow A. The advantages and limitations of trait analysis with gwas: a review. Plant Methods. 2013;9(1):29.

20. Qian L, Hickey LT, Stahl A, Werner CR, Hayes B, Snowdon RJ, Voss-Fels KP. Exploring and harnessing haplotype diversity to improve yield stability in crops. Front Plant Sci. 2017;8:1534.

21. Appels R, Eversole K, Feuillet C, Keller B, Rogers J, Stein N, Pozniak CJ, Choulet F, Distelfeld A, Poland J, et al. Shifting the limits in wheat research and breeding using a fully annotated reference genome. Science. 2018;361(6403):7191.

22. Allen AM, Winfield MO, Burridge AJ, Downie RC, Benbow HR, Barker GL, Wilkinson PA, Coghill J, Waterfall C, Davassi A, et al. Characterization of a wheat breeders' array suitable for high-throughput snp genotyping of global accessions of hexaploid bread wheat (triticum aestivum). Plant Biotechnol J. 2017;15(3):390-401.

23. Mirabella N, Abbate P, Alonso M, Panelo J, Pontaroli A. Identifying traits at crop maturity and models for estimation of lodging susceptibility in bread wheat. Crop Pasture Sci. 2019;70(2):95-106.

24. Engledow F, Wadham S. Plant characters on yield. J Agric Sci. 1923;13: 390-439.

25. Fischer RA. Wheat. potential productivity of field crops under different environments: International Rice Research Institute, Los Baños, Philippines; 1984, pp. 129-54.

26. Abbate PE, Pontaroli AC, Lázaro L, Gutheim F. A method of screening for spike fertility in wheat. J Agric Sci. 2013;151(3):322-30.

27. González F, Terrile II, Falcón M. Spike fertility and duration of stem elongation as promising traits to improve potential grain number (and yield): variation in modern argentinean wheats. Crop Sci. 2011;51(4): 1693-702.

28. Mirabella N, Abbate P, Ramirez I, Pontaroli A. Genetic variation for wheat spike fertility in cultivars and early breeding materials. J Agric Sci. 2016;154(1):13-22.

29. Martino DL, Abbate PE, Cendoya MG, Gutheim F, Mirabella NE, Pontaroli AC. Wheat spike fertility: inheritance and relationship with spike yield components in early generations. Plant Breeding. 2015;134(3):264-70.

30. Fischer R. Wheat physiology: a review of recent developments. Crop Pasture Sci. 2011;62(2):95-114

31. François $O$. Running structure-like population genetic analyses with r. $R$ Tutor Popul Genet U Grenoble-Alpes. 2016;1-9.

32. Vanzetti LS, Yerkovich N, Chialvo E, Lombardo L, Vaschetto L, Helguera M. Genetic structure of argentinean hexaploid wheat germplasm. Genet Mol Biol. 2013;36(3):391-9.

33. Gomez D, Vanzetti L, Helguera M, Lombardo L, Fraschina J, Miralles DJ. Effect of vrn-1, ppd-1 genes and earliness per se on heading time in argentinean bread wheat cultivars. Field Crops Res. 2014;158:73-81.

34. Mater Y, Baenziger S, Gill K, Graybosch R, Whitcher L, Baker C, Specht J, Dweikat I. Linkage mapping of powdery mildew and greenbug resistance genes on recombinant 1RS from 'Amigo' and 'Kavkaz' wheat-rye translocations of chromosome 1RS.1 AL.. Genome. 2004;47(2):292-8. https://doi.org/10.1139/g03-101.

35. Graybosch RA. Uneasy unions: Quality effects of rye chromatin transfers to wheat. J Cereal Sci. 2001;33(1):3-16. https://doi.org/10.1006/jcrs.2000. 0336.

36. Singh S, Vikram P, Sehgal D, Burgueño J, Sharma A, Singh SK, Sansaloni $C P$, Joynson R, Brabbs T, Ortiz C, et al. Harnessing genetic potential of wheat germplasm banks through impact-oriented-prebreeding for future food and nutritional security. Sci Rep. 2018;8(1):12527.

37. Bevan MW, Uauy C, Wulff BB, Zhou J, Krasileva K, Clark MD. Genomic innovation for crop improvement. Nature. 2017;543(7645):346.

38. Slafer GA, Elia M, Savin R, García GA, Terrile II, Ferrante A, Miralles DJ, González FG. Fruiting efficiency: an alternative trait to further rise wheat yield. Food Energy Secur. 2015;4(2):92-109.

39. Terrile II, Miralles DJ, González FG. Fruiting efficiency in wheat (triticum aestivum I): Trait response to different growing conditions and its relation to spike dry weight at anthesis and grain weight at harvest. Field Crops Res. 2017;201:86-96.
40. Guo Z, Chen D, Alqudah AM, Röder MS, Ganal MW, Schnurbusch T. Genome-wide association analyses of 54 traits identified multiple loci for the determination of floret fertility in wheat. New Phytol. 2017;214(1): 257-70.

41. Juliana RP, Singh P, Singh PK, Poland JA, Bergstrom GC, Huerta-Espino J, Bhavani S, Crossa J, Sorrells ME. Genome-wide association mapping for resistance to leaf rust, stripe rust and tan spot in wheat reveals potential candidate genes. Theor Appl Genet. 2018;131:1-18.

42. Sakuma S, Golan G, Guo Z, Ogawa T, Tagiri A, Sugimoto K, Bernhardt N, Brassac J, Mascher M, Hensel G, et al. Unleashing floret fertility in wheat through the mutation of a homeobox gene. Proc Natl Acad Sci. 2019;116(11):5182-7.

43. Su Z, Hao C, Wang L, Dong Y, Zhang X. Identification and development of a functional marker of tagw2 associated with grain weight in bread wheat (triticum aestivum I.) Theor Appl Genet. 2011;122(1):211-23.

44. Simmonds J, Scott P, Brinton J, Mestre TC, Bush M, Del Blanco A, Dubcovsky J, Uauy C. A splice acceptor site mutation in tagw2-a1 increases thousand grain weight in tetraploid and hexaploid wheat through wider and longer grains. Theor Appl Genet. 2016;129(6): 1099-112.

45. Ma L, Li T, Hao C, Wang Y, Chen $X$, Zhang $X$. Ta gs 5-3a, a grain size gene selected during wheat improvement for larger kernel and yield. Plant Biotechnol J. 2016;14(5):1269-80.

46. Liu J, Xu Z, Fan X, Zhou Q, Cao J, Wang F, Ji G, Yang L, Feng B, Wang T. A genome-wide association study of wheat spike related traits in china. Front Plant Sci. 2018;9:1584

47. Beales J, Turner A, Griffiths S, Snape JW, Laurie DA. A pseudo-response regulator is misexpressed in the photoperiod insensitive ppd-d1a mutant of wheat (triticum aestivum I.) Theor Appl Genet. 2007;1 15(5):721-33.

48. Zikhali M, Wingen LU, Leverington-Waite M, Specel S, Griffiths S. The identification of new candidate genes triticum aestivum flowering locus t3-b1 (taft3-b1) and target of eat1 (tatoe1-b1) controlling the short-day photoperiod response in bread wheat. Plant Cell Environ. 2017;40(11): 2678-90.

49. Mizuno N, Kinoshita M, Kinoshita S, Nishida H, Fujita M, Kato K, Murai K, Nasuda S. Loss-of-function mutations in three homoeologous phytoclock 1 genes in common wheat are associated with the extra-early flowering phenotype. PLOS ONE. 2016;11(10):0165618.

50. Yan L, Helguera M, Kato K, Fukuyama S, Sherman J, Dubcovsky J. Allelic variation at the vrn-1 promoter region in polyploid wheat. Theor Appl Genet. 2004;109(8):1677-86.

51. Fu D, Szúcs P, Yan L, Helguera M, Skinner JS, Von Zitzewitz J, Hayes PM Dubcovsky J. Large deletions within the first intron in vrn-1 are associated with spring growth habit in barley and wheat. Mol Genet Genomics. 2005;273(1):54-65.

52. Díaz A, Zikhali M, Turner AS, Isaac $P$, Laurie DA. Copy number variation affecting the photoperiod-b1 and vernalization-a1 genes is associated with altered flowering time in wheat (triticum aestivum). PLOS ONE. 2012;7(3):33234.

53. Ellis M, Spielmeyer W, Gale K, Rebetzke G, Richards R. "perfect" markers for the rht-b $1 \mathrm{~b}$ and rht-d $1 \mathrm{~b}$ dwarfing genes in wheat. Theor Appl Genet. 2002;105(6-7):1038-42.

54. Ellis M, Rebetzke G, Azanza F, Richards R, Spielmeyer W. Molecular mapping of gibberellin-responsive dwarfing genes in bread wheat. Theor Appl Genet. 2005;111(3):423-30.

55. Mo Y, Vanzetti LS, Hale I, Spagnolo EJ, Guidobaldi F, Al-Oboudi J, Odle $\mathrm{N}$, Pearce S, Helguera M, Dubcovsky J. Identification and characterization of rht25, a locus on chromosome arm 6as affecting wheat plant height, heading time, and spike development. Theor Appl Genet. 2018;131(10):2021-35.

56. Ford BA, Foo E, Sharwood R, Karafiatova M, Vrána J, MacMillan C, Nichols DS, Steuernagel B, Uauy C, Doležel J, et al. Rht18 semidwarfism in wheat is due to increased ga 2-oxidasea9 expression and reduced ga content. Plant Physiol. 2018;177(1):168-80.

57. Wang H, Smith KP, Combs E, Blake T, Horsley RD, Muehlbauer GJ. Effect of population size and unbalanced data sets on qtl detection using genome-wide association mapping in barley breeding germplasm. Theor Appl Genet. 2012;124(1):111-24.

58. Zikhali M, Wingen LU, Griffiths S. Delimitation of the earliness per se d1 (eps-d1) flowering gene to a subtelomeric chromosomal deletion in bread wheat (triticum aestivum). J Exp Bot. 2015;67(1):287-99. 
59. Schachermayr G, Feuillet C, Keller B. Molecular markers for the detection of the wheat leaf rust resistance gene $\operatorname{lr} 10$ in diverse genetic backgrounds. Mol Breed. 1997;3(1):65-74.

60. Lagudah E, McFadden H, Singh R, Huerta-Espino J, Bariana H, Spielmeyer W. Molecular genetic characterization of the Ir34/yr18 slow rusting resistance gene region in wheat. Theor Appl Genet. 2006;114(1):21-30.

61. Schachermayr G, Messmer M, Feuillet C, Winzeler H, Winzeler M, Keller B. Identification of molecular markers linked to the agropyron elongatum-derived leaf rust resistance gene Ir24 in wheat. Theor Appl Genet. 1995;90(7-8):982-90.

62. He X, He Z, Zhang L, Sun D, Morris C, Fuerst E, Xia X. Allelic variation of polyphenol oxidase (ppo) genes located on chromosomes $2 \mathrm{a}$ and $2 \mathrm{~d}$ and development of functional markers for the ppo genes in common wheat. Theor Appl Genet. 2007;115(1):47-58.

63. Himi E, Maekawa M, Miura H, Noda K. Development of pcr markers for tamyb10 related to $r-1$, red grain color gene in wheat. Theor Appl Genet. 2011;122(8):1561-76.

64. Butow B, Gale K, Ikea J, Juhasz A, Bedö Z, Tamas L, Gianibelli M. Dissemination of the highly expressed bx7 glutenin subunit (glu-b1al allele) in wheat as revealed by novel pcr markers and rp-hplc. Theor Appl Genet. 2004;109(7):1525-35.

65. Vanzetti LS, Pflüger LA, Rodríguez-Quijano M, Carrillo JM, Helguera M. Genetic variability for waxy genes in argentinean bread wheat germplasm. Electron J Biotechnol. 2009;12(1):4-5.

66. Yang Y, Zhao X, Xia L, Chen X, Xia X, Yu Z, He Z, Röder M. Development and validation of a viviparous-1 sts marker for pre-harvest sprouting tolerance in chinese wheats. Theor Appl Genet. 2007;115(7):971-80.

67. Zhang W, Gianibelli M, Rampling L, Gale K. Characterisation and marker development for low molecular weight glutenin genes from glu-a3 alleles of bread wheat (triticum aestivum. I). Theor Appl Genet. 2004;108(7):1409-19.

68. Gautier M-F, Aleman M-E, Guirao A, Marion D, Joudrier P. Triticum aestivum puroindolines, two basic cystine-rich seed proteins: cdna sequence analysis and developmental gene expression. Plant Mol Biol. 1994;25(1):43-57.

69. Pflüger L, D'Ovidio R, Margiotta B, Pena R, Mujeeb-Kazi A, Lafiandra D. Characterisation of high-and low-molecular weight glutenin subunits associated to the $\mathrm{d}$ genome of aegilops tauschii in a collection of synthetic hexaploid wheats. Theor Appl Genet. 2001;103(8):1293-301.

70. Barrett JC, Fry B, Maller J, Daly MJ. Haploview: analysis and visualization of Id and haplotype maps. Bioinformatics. 2004;21(2):263-5.

71. Lipka AE, Tian F, Wang Q, Peiffer J, Li M, Bradbury PJ, Gore MA, Buckler ES, Zhang Z. Gapit: genome association and prediction integrated tool. Bioinformatics. 2012;28(18):2397-9.

72. Zadoks JC, Chang TT, Konzak CF. A decimal code for the growth stages of cereals. Weed Res. 1974;14(6):415-21.

73. Crescente JM, Guidobaldi F, Demichelis M, Formica MB, Helguera M, Vanzetti LS. Phenobook: an open source software for phenotypic data collection. GigaScience. 2017;6(4):1-5.

74. Wang Q, Tian F, Pan Y, Buckler ES, Zhang Z. A super powerful method for genome wide association study. PLoS ONE. 2014;9(9):107684.

75. Zhang J, Gizaw SA, Bossolini E, Hegarty J, Howell T, Carter AH, Akhunov E, Dubcovsky J. Identification and validation of qtl for grain yield and plant water status under contrasting water treatments in fall-sown spring wheats. Theor Appl Genet. 2018;131:1-19.

\section{Publisher's Note}

Springer Nature remains neutral with regard to jurisdictional claims in published maps and institutional affiliations.

Ready to submit your research? Choose BMC and benefit from:

- fast, convenient online submission

- thorough peer review by experienced researchers in your field

- rapid publication on acceptance

- support for research data, including large and complex data types

- gold Open Access which fosters wider collaboration and increased citations

- maximum visibility for your research: over $100 \mathrm{M}$ website views per year

At $\mathrm{BMC}$, research is always in progress.

Learn more biomedcentral.com/submissions 